\title{
Consistent truncation and de Sitter space from gravitational instantons
}

\section{Robin Terrisse and Dimitrios Tsimpis}

Institut de Physique Nucléaire de Lyon, Université de Lyon, UCBL, UMR 5822, CNRS/IN2P3, 4 rue Enrico Fermi, 69622 Villeurbanne Cedex, France

E-mail: terrisse@ipnl.in2p3.fr, tsimpis@ipnl.in2p3.fr

ABSTRACT: We construct a four-dimensional consistent truncation to the bosonic part of the universal sector of Calabi-Yau IIA compactification (i.e. the gravity multiplet, one vectormultiplet, and one hypermultiplet) in the presence of background flux and fermionic condensates generated by gravitational instantons. The condensates are controlled by the ratio of the characteristic length of the Calabi-Yau to the string length, and can be finetuned to be dominant in a region of large volume and small string coupling. The consistent truncation admits de Sitter solutions supported by the condensates, subject to certain validity conditions that we discuss.

KEYWORDS: Flux compactifications, Superstring Vacua

ARXIV EPRINT: 1903.10504 


\section{Contents}

1 Introduction 1

2 Review of IIA reduction on CY 3

2.1 Derivative corrections 4

3 Consistent truncation $\quad 6$

3.1 Action and equations of motion 6

$\begin{array}{lll}3.2 & \text { Consistent truncation without condensates } & 8\end{array}$

$\begin{array}{ll}3.3 \text { Consistent truncation with condensates } & 12\end{array}$

$\begin{array}{lll}3.4 \text { Vacua } & 15\end{array}$

$\begin{array}{lll}4 & \text { Discussion } & 17\end{array}$

$\begin{array}{lr}\text { A Conventions } & 18\end{array}$

$\begin{array}{lr}\text { B ALE instantons } & 19\end{array}$

C Gravitino condensates in $4 \mathrm{~d} \mathcal{N}=1$ supergravity 21

\section{Introduction}

Within the framework of critical ten-dimensional superstring theories, it has proven very difficult to obtain controlled scenarios leading to a low-energy effective theory with a positive cosmological constant (CC) or dark energy, see $[1,2]$ for recent reviews. On rather general grounds, the presence of de Sitter vacua within the limit of classical two-derivative supergravity is excluded $[3,4]$, thus one is led to include quantum corrections in order to evade the no-go theorem. ${ }^{1}$ One such quantum effect is fermionic condensation, which is known to occur in supersymmetric Yang-Mills theories [9].

The vacuum expectation value (VEV) of all single fermions should vanish in order to preserve the symmetries of a maximally-symmetric vacuum of the theory, however nonvanishing quadratic or quartic fermion VEV's (condensates) may still be generated by nonperturbative effects such as instantons. Fermionic condensates have thus the potential to generate a positive contribution to the CC. Within the framework of the ten-dimensional superstrings, most studies have focused on gaugino condensation in the heterotic theory [10-17], which however does not seem to allow a positive CC [18]. Recent results [19, 20] indicate that the situation is more encouraging within the framework of the IIA superstring - which appears to allow the generation of a positive CC by fermionic condensates, at least in principle.

\footnotetext{
${ }^{1}$ The no-go theorem might also be circumvented at the classical level by including orientifold sources (see [5] for a recent proposal), although this scenario is also subject to stringent constraints [6-8].
} 
In the functional integration over metrics approach to quantum gravity [21], the gravitino condensates arise from saddle points of the $4 \mathrm{~d}$ action corresponding to gravitational instantons. These are noncompact asymptotically locally Euclidean (ALE) spaces with selfdual Riemann curvature and thus vanishing Einstein action [22]. Although other saddle points may exist in the presence of matter fields [23], they would have positive Euclidean action. $^{2}$ Thus ALE spaces are expected to capture the dominant instanton contributions in the path integral approach of quantum gravity.

Going beyond the two-derivative approximation of the $4 \mathrm{~d}$ effective action, the gravitational instantons give a positive contribution to the action at the four-derivative order. Among the ALE spaces, the one with the minimal four-derivative action is the EguchiHanson (EH) gravitational instanton [28]. In the EH background there are two positivechirality spin-3/2 zero modes of the Dirac operator, and no spin- $1 / 2$ zero modes, thus giving rise to a nonvanishing gravitino bilinear condensate in four dimensions at one loop in the $4 \mathrm{~d}$ gravitational coupling $[29,30]$. The quartic gravitino VEV's receive contributions from ALE instantons with higher Hirzebruch signature. Since ALE spaces do not support spin-1/2 zero modes, no dilatino condensates are generated.

The aim of the present paper is to study gravitino condensation in the IIA theory compactified on Calabi-Yau (CY) threefolds. To that end we construct a 4d consistent truncation capturing the bosonic part of the universal sector of CY IIA compactification (i.e. the gravity multiplet, one vectormultiplet, and one hypermultiplet) in the presence of background flux and gravitino condensates generated by ALE instantons.

In the limit of vanishing flux and condensates, our construction reduces to the universal bosonic sector of the effective action of IIA CY compactifications (at the two-derivative order) thus proving that the latter is also a consistent truncation. In the presence of nonvanishing flux and fermion condensates, the result should be thought of as a subsector of the $4 \mathrm{~d}$ effective action, in the limit where the masses induced by the flux and/or the condensate are sufficiently smaller than the Kaluza-Klein (KK) scale.

The condensates are controlled by the ratio of the characteristic length of the CY to the string length, and can be fine-tuned to be dominant in a region of large volume and small string coupling. The consistent truncation admits de Sitter solutions supported by the condensates, subject to certain validity conditions that we discuss.

The plan of the remainder of the paper is as follows. Section 2 includes a brief review of the reduction of IIA on CY at the two-derivative level, in the absence of flux and condensates. Higher-order derivative corrections in the $4 \mathrm{~d}$ action are discussed in section 2.1. The consistent truncation to the universal sector is contained in section 3: in section 3.2 we construct the truncation in the presence of background flux. A further extension to include gravitino condensates is constructed in section 3.3. Maximally-symmetric vacua thereof are discussed in section 3.4. Section 4 discusses the conditions of validity of our results, and some open directions. Appendix A discusses our spinor conventions. A brief review of ALE spaces is given in section B. The general form of the gravitino condensates,

\footnotetext{
${ }^{2}$ This follows from the positive action conjecture [21], which in its turn can be seen to follow from the positive energy theorem [24-27].
} 
generated by ALE gravitational instantons in the context of $4 \mathrm{~d} \mathcal{N}=1$ supergravity, is reviewed in appendix $\mathrm{C}$.

\section{Review of IIA reduction on $\mathrm{CY}$}

To establish notation and conventions, let us briefly review the reduction of IIA on CY at the two-derivative level, in the absence of flux and condensates. As is well known, the KK reduction of (massless) IIA supergravity around the fluxless $\mathbb{R}^{1,3} \times Y$ vacuum results in a $4 \mathrm{~d}$ $\mathcal{N}=2$ supergravity, whose bosonic sector consists of one gravity multiplet (containing the metric and one vector), $h^{1,1}$ vector multiplets (each of which consists of one vector and two real scalars) and $h^{2,1}+1$ hypermultiplets (each of which contains four real scalars), where $h^{p, q}$ are the Hodge numbers of the CY threefold $Y$. The $2 h^{1,1}$ real scalars $\left(v^{A}, \chi^{A}\right)$ in the vector multiplets come from the NS-NS $B$ field and deformations of the metric of the form,

$$
B=\beta(x)+\sum_{A=1}^{h^{1,1}} \chi^{A}(x) e^{A}(y) ; \quad i \delta g_{a \bar{b}}=\sum_{A=1}^{h^{1,1}} v^{A}(x) e_{a \bar{b}}^{A}(y)
$$

where $\beta$ is a two-form in $\mathbb{R}^{1,3} ;\left\{e_{a \bar{b}}^{A}(y), A=1, \ldots, h^{1,1}\right\}$ is a basis of harmonic $(1,1)$-forms on the $\mathrm{CY}$, and $x, y$ are coordinates of $\mathbb{R}^{1,3}, Y$ respectively; we have introduced holomorphic, antiholomorphic internal indices from the beginning of the latin alphabet: $a=1, \ldots, 3$, $\bar{b},=1, \ldots, 3$, respectively. Since every CY has a Kähler form (which can be expressed as a linear combination of the basis $(1,1)$-forms), there is a always at least one vector multiplet (which may be called "universal", in that it exists for any CY compactification) whose scalars consist of the volume modulus $v$ and one scalar $\chi$.

The $2\left(h^{2,1}+1\right)$ complex scalars of the hypermultiplets, and the $h^{1,1}+1$ vectors of the gravity and the vectormultiplets arise as follows: from the one- and three-form RR potentials $C_{1}, C_{3}$ and the complex-structure deformations of the metric, ${ }^{3}$

$$
\begin{aligned}
\delta g_{\bar{a} \bar{b}} & =\sum_{\alpha=1}^{h^{2,1}} \zeta^{\alpha}(x) \Omega_{\bar{a}}^{* c d} \Phi_{c d \bar{b}}^{\alpha}(y) ; \quad C_{1}=\alpha(x) ; \\
C_{3} & =-\frac{1}{2}\left(\xi(x) \operatorname{Im} \Omega+\xi^{\prime}(x) \operatorname{Re} \Omega\right)+\sum_{A=1}^{h^{1,1}} \gamma^{A}(x) \wedge e^{A}(y)+\left(\sum_{\alpha=1}^{h^{2,1}} \xi^{\alpha}(x) \Phi^{\alpha}(y)+\text { c.c. }\right),
\end{aligned}
$$

where $\Omega(y)$ is the holomorphic threeform of the CY and $\left\{\Phi_{a b \bar{c}}^{\alpha}(y), \alpha=1, \ldots, h^{2,1}\right\}$ is basis of harmonic $(2,1)$ forms on the CY, we obtain the complex scalars $\left(\zeta^{\alpha}, \xi^{\alpha}\right)$ and the vectors $\left(\alpha, \gamma^{A}\right)$. Moreover the real scalars $\left(\xi, \xi^{\prime}\right)$ together with the dilaton $\phi$ and the axion $b$ combine into one universal hypermultiplet. Recall that if $h$ is the $4 \mathrm{~d}$ component of the NSNS three-form,

$$
h=\mathrm{d} \beta,
$$

the axion $b$ is given schematically by $\mathrm{d} b \sim \star_{4} h$ (the precise relation is eq. (3.22) below).

\footnotetext{
${ }^{3}$ The right-hand side of the first equation of $(2.2)$ can be seen to be automatically symmetric in its two free indices.
} 
In summary, the universal bosonic sector of the $4 \mathrm{~d} \mathcal{N}=2$ supergravity arising from IIA compactification on $Y$ contains the metric and the vector of the gravity multiplet $\left(g_{\mu \nu}, \alpha\right)$, the vector and the scalars of one vectormultiplet $(\gamma, v, \chi)$, and the scalars of the universal hypermultiplet $\left(\xi, \xi^{\prime}, \phi, b\right)$.

\subsection{Derivative corrections}

Four-derivative corrections to the $4 \mathrm{~d}$ effective action resulting from compactification of the IIA superstring on CY threefolds have been known since [31]. More recently they have been computed in [32] (see also [33]), from compactification of certain known terms of the ten-dimensional IIA tree-level and one-loop superstring effective action at order $\alpha^{\prime 3}$. The authors of that reference take into account the graviton and $B$-field eight-derivative terms given in $[34,35]$, but neglect e.g. the dilaton derivative couplings and RR couplings of the form $R^{2}(\partial F)^{2}$ and $\partial^{4} F^{4}$ calculated in [36]. Furthermore [32] neglects loop corrections from massive KK fields. ${ }^{4}$

In a low-energy expansion, the $4 \mathrm{~d}$ effective action takes the schematic form [38],

$$
2 \kappa^{2} S=\int \mathrm{d} x^{4} \sqrt{g}\left(R+\beta_{1} \alpha^{\prime} R^{2}+\beta_{2} \alpha^{\prime 2} R^{3}+\beta_{3} \alpha^{\prime 3} R^{4}\right),
$$

where $\kappa$ is the four-dimensional gravitational constant, and a Weyl transformation must be performed to bring the action to the $4 \mathrm{~d}$ Einstein frame. ${ }^{5}$ Moreover each coefficient in the series can be further expanded in the string coupling to separate the tree-level from the one-loop contributions. Although all the higher-derivative terms in (2.4) descend from the eight-derivative ten-dimensional $\alpha^{\prime 3}$-corrections, they correspond to different orders of the $4 \mathrm{~d}$ low-energy expansion. Indeed if $l_{s}=2 \pi \sqrt{\alpha^{\prime}}, l_{4 d}$ and $l_{Y}$ are the string length, the fourdimensional low-energy wavelength and the characteristic length of $Y$ respectively, we have,

$$
l_{s}^{2} \ll l_{Y}^{2} \ll l_{4 d}^{2} .
$$

Moreover the term with coefficient $\beta_{n}$ in (2.4) is of order,

$$
\left(\frac{l_{s}}{l_{4 d}}\right)^{2 n}\left(\frac{l_{s}}{l_{Y}}\right)^{6-2 n} ; n=1,2,3
$$

relative to the Einstein term, so that the $n=1$ term dominates the $n=2,3$ terms in (2.4).

The ten-dimensional IIA supergravity (two-derivative) action admits solutions without flux of the form $\mathbb{R}^{1,3} \times Y$, where $Y$ is of $\mathrm{SU}(3)$ holonomy (which for our purposes we take to be a compact CY). A sigma model argument [39] shows that this background can

\footnotetext{
${ }^{4}$ Presumably the KK loop corrections are subleading and vanish in the large-volume limit (see however [37] for an exception to this statement). At any rate these corrections are dependent on the specific $\mathrm{CY}$ and at the moment can only be computed on a case-by-case basis, e.g. around the orbifold limit where the CY reduces to $T^{6} / \Gamma$ with $\Gamma$ a discrete group. Winding modes are heavier than KK modes in a regime where (2.5) holds.

${ }^{5}$ As emphasized in [32], in computing the $4 \mathrm{~d}$ effective action the compactification must be performed around the solution to the $\alpha^{\prime}$-corrected equations of motion. This procedure can thus generate $\alpha^{\prime}$-corrections also from the compactification of the ten-dimensional Einstein term.
} 
be promoted to a solution to all orders in $\alpha^{\prime}$, provided the metric of $Y$ is appropriately corrected at each order in such a way that it remains Kähler. ${ }^{6}$ Indeed [32] confirms this to order $\alpha^{\prime 3}$ and derives the explicit corrections to the dilaton and the metric, which is deformed away from Ricci-flatness at this order. Their derivation remains valid for backgrounds of the form $M_{4} \times Y$, where $M_{4}$ is any Ricci-flat four-dimensional space.

Within the framework of the effective $4 \mathrm{~d}$ theory, nonperturbative gravitational instanton corrections arise from vacua of the form $M_{4} \times Y$, where $M_{4}$ is an ALE space. These instanton contributions are weighted by a factor $\exp \left(-S_{0}\right)$, where $S_{0}$ is the 4 d effective action evaluated on the solution $M_{4} \times Y$. Subject to the limitations discussed above, and taking into account the Ricci-flatness of the metric of $M_{4}$, the IIA $4 \mathrm{~d}$ effective action of [32] reduces to,

$$
2 \kappa^{2} S_{0}=\beta_{1} \alpha^{\prime} \int_{M_{4}} \mathrm{~d} x^{4} \sqrt{g} R_{\kappa \lambda \mu \nu} R^{\kappa \lambda \mu \nu},
$$

where in the conventions of $[32],{ }^{7}$

$$
\kappa^{2}=\pi \alpha^{\prime} ; \quad M_{\mathrm{P}}=2 \sqrt{\pi} l_{s}^{-1},
$$

with $M_{\mathrm{P}}=\kappa^{-1}$ the (reduced) $4 \mathrm{~d}$ Planck mass and $\beta_{1}$ given by,

$$
l_{s}^{6} \beta_{1}=2^{9} \pi^{4} \alpha^{\prime 2} \int_{Y} c_{2} \wedge J
$$

where $c_{2}$ is the second Chern class of $Y$. For a generic Kähler manifold we have,

$$
c_{2} \wedge J=\frac{1}{32 \pi^{2}}\left(R_{m n k l}^{2}-\mathfrak{R}_{m n}^{2}+\frac{1}{4} \mathfrak{R}^{2}\right) \operatorname{vol}_{6},
$$

where we have adopted real notation and defined $\mathfrak{R}_{m n}:=R_{m n k l} J^{k l}, \mathfrak{R}:=\mathfrak{R}_{m n} J^{m n}$. The contractions are taken with respect to the metric compatible with the Kähler form $J$ and the connection of the Riemann tensor.

The information about $Y$ enters the $4 \mathrm{~d}$ effective action through the calculation of $\beta_{1}$. Since $\beta_{1}$ multiplies a term which is already a higher-order correction, it suffices to evaluate it in the CY limit (for which $\mathfrak{R}_{m n}$ vanishes). We thus obtain,

$$
\beta_{1}=\frac{1}{\pi^{2} l_{s}^{2}} \int_{Y} \mathrm{~d}^{6} x \sqrt{g} R_{m n k l}^{2}>0 .
$$

Therefore the leading instanton contribution comes from the ALE space which minimizes the integral in (2.7). This is the EH space [41], cf. (B.5), so that,

$$
S_{0}=\frac{24}{\pi l_{s}^{2}} \int_{Y} \mathrm{~d}^{6} x \sqrt{g} R_{m n k l}^{2}>0 .
$$

\footnotetext{
${ }^{6}$ It should be possible to generalize the sigma-model argument of [39] to the case of backgrounds of the form $M_{4} \times Y$, where $M_{4}$ is an ALE space, along the lines of [40].

${ }^{7}$ The ten-dimensional gravitational constant of [32] $2 \kappa_{10}^{2}=(2 \pi)^{7} \alpha^{\prime 4}$, cf. (2.4) therein, is related to the four-dimensional one via $\kappa^{2}=\kappa_{10}^{2} / l_{s}^{6}$. Note in particular that eqs. (4.9) and (4.19) of that reference are given in units where $l_{s}=2 \pi \sqrt{\alpha^{\prime}}=1$ : to reinstate engineering dimensions one must multiply with the appropriate powers of $l_{s}$. The $4 \mathrm{~d}$ Einstein term in (2.4) has been canonically normalized via a Weyl transformation of the $4 \mathrm{~d}$ metric. This affects the relative coefficient between two- and four-derivative terms in the action: note in particular that the right-hand side of (2.7) is invariant under Weyl transformations. We thank Kilian Mayer for clarifying to us the conventions of [32].
} 
Note that $S_{0}$ does not depend on the dilaton: this is related to the fact that, starting from an action of the form $\int \mathrm{d}^{4} x \sqrt{g}\left(e^{-2 \phi} R+\beta_{1} \alpha^{\prime} R_{\mu \nu \rho \sigma}^{2}\right)$, the dilaton exponential can be absorbed by a Weyl transformation of the form $g_{\mu \nu} \rightarrow e^{2 \phi} g_{\mu \nu}$, cf. footnote 7 . Therefore we have,

$$
S_{0}=c\left(\frac{l_{Y}}{l_{s}}\right)^{2}
$$

with $c$ a positive number of order one.

\section{Consistent truncation}

In [20] we presented a universal consistent truncation on Nearly-Kähler and CY manifolds in the presence of dilatino condensates. As it turns out, this consistent truncation captures only part of the universal scalar sector of the $\mathcal{N}=2$ low-energy effective supergravity obtained from IIA theory compactified on CY threefolds. Therefore we must extend the ansatz of [20] to include the "missing" fields and also to take into account the gravitino condensates.

\subsection{Action and equations of motion}

In [19] the quartic dilatino terms of all (massive) IIA supergravities [42-46] were determined in the ten-dimensional superspace formalism of [47], and were found to agree with [42]. As follows from the result of [47], the quartic fermion terms are common to all IIA supergravities (massive or otherwise). In the following we will complete Romans supergravity (whose quartic fermion terms were not computed in [45]) by adding the quartic gravitino terms given in [42]. Furthermore we will set the dilatino to zero. Of course this would be inconsistent in general, since the dilatino couples linearly to gravitino terms. Here this does not lead to an inconsistency in the equations of motion, since we are ultimately interested in a maximally-symmettric vacuum, in which linear and cubic fermion VEV's vanish.

In the conventions of $[19,20]$, upon setting the dilatino to zero, the action of Romans supergravity reads,

$$
\begin{aligned}
S=S_{b}+\frac{1}{2 \kappa_{10}^{2}} \int \mathrm{d}^{10} x \sqrt{g} & 2\left(\tilde{\Psi}_{M} \Gamma^{M N P} \nabla_{N} \Psi_{P}\right)+\frac{1}{2} e^{5 \phi / 4} m\left(\tilde{\Psi}_{M} \Gamma^{M N} \Psi_{N}\right) \\
& -\frac{1}{2 \cdot 2 !} e^{3 \phi / 4} F_{M_{1} M_{2}}\left(\tilde{\Psi}^{M} \Gamma_{[M} \Gamma^{M_{1} M_{2}} \Gamma_{N]} \Gamma_{11} \Psi^{N}\right) \\
& -\frac{1}{2 \cdot 3 !} e^{-\phi / 2} H_{M_{1} \ldots M_{3}}\left(\tilde{\Psi}^{M} \Gamma_{[M} \Gamma^{M_{1} \ldots M_{3}} \Gamma_{N]} \Gamma_{11} \Psi^{N}\right) \\
& \left.+\frac{1}{2 \cdot 4 !} e^{\phi / 4} G_{M_{1} \ldots M_{4}}\left(\tilde{\Psi}^{M} \Gamma_{[M} \Gamma^{M_{1} \ldots M_{4}} \Gamma_{N]} \Psi^{N}\right)+L_{\Psi^{4}}\right\}
\end{aligned}
$$

where $\Psi_{M}$ is the gravitino; $S_{b}$ denotes the bosonic sector,

$$
\begin{aligned}
S_{b}=\frac{1}{2 \kappa_{10}^{2}} \int \mathrm{d}^{10} x \sqrt{g}( & -R+\frac{1}{2}(\partial \phi)^{2}+\frac{1}{2 \cdot 2 !} e^{3 \phi / 2} F^{2} \\
& \left.+\frac{1}{2 \cdot 3 !} e^{-\phi} H^{2}+\frac{1}{2 \cdot 4 !} e^{\phi / 2} G^{2}+\frac{1}{2} m^{2} e^{5 \phi / 2}\right)+\mathrm{CS},
\end{aligned}
$$


and CS is the Chern-Simons term. There are 24 quartic gravitino terms as given in [42], denoted $L_{\Psi^{4}}$ in (3.1). Of these only four can have a nonvanishing VEV in an ALE space: they are discussed in more detail in section 3.3.

We emphasize that the action (3.1) should be regarded as a book-keeping device whose variation with respect to the bosonic fields gives the correct bosonic equations of motion in the presence of gravitino condensates. Furthermore, the fermionic equations of motion are trivially satisfied in the maximally-symmetric vacuum. The (bosonic) equations of motion (EOM) following from (3.1) are as follows:

\section{Dilaton EOM,}

$$
\begin{aligned}
0= & -\nabla^{2} \phi+\frac{3}{8} e^{3 \phi / 2} F^{2}-\frac{1}{12} e^{-\phi} H^{2}+\frac{1}{96} e^{\phi / 2} G^{2}+\frac{5}{4} m^{2} e^{5 \phi / 2} \\
& +\frac{5}{8} e^{5 \phi / 4} m\left(\tilde{\Psi}_{M} \Gamma^{M N} \Psi_{N}\right) \\
& -\frac{3}{16} e^{3 \phi / 4} F_{M_{1} M_{2}}\left(\tilde{\Psi}^{M} \Gamma_{[M} \Gamma^{M_{1} M_{2}} \Gamma_{N]} \Gamma_{11} \Psi^{N}\right) \\
& +\frac{1}{24} e^{-\phi / 2} H_{M_{1} \ldots M_{3}}\left(\tilde{\Psi}^{M} \Gamma_{[M} \Gamma^{M_{1} \ldots M_{3}} \Gamma_{N]} \Gamma_{11} \Psi^{N}\right) \\
& +\frac{1}{192} e^{\phi / 4} G_{M_{1} \ldots M_{4}}\left(\tilde{\Psi}^{M} \Gamma_{[M} \Gamma^{M_{1} \ldots M_{4}} \Gamma_{N]} \Psi^{N}\right)
\end{aligned}
$$

\section{Einstein EOM,}

$$
\begin{aligned}
& R_{M N}=\frac{1}{2} \partial_{M} \phi \partial_{N} \phi+\frac{1}{16} m^{2} e^{5 \phi / 2} g_{M N}+\frac{1}{4} e^{3 \phi / 2}\left(2 F_{M N}^{2}-\frac{1}{8} g_{M N} F^{2}\right) \\
& +\frac{1}{12} e^{-\phi}\left(3 H_{M N}^{2}-\frac{1}{4} g_{M N} H^{2}\right)+\frac{1}{48} e^{\phi / 2}\left(4 G_{M N}^{2}-\frac{3}{8} g_{M N} G^{2}\right) \\
& +\frac{1}{24} e^{\phi / 4} G_{(M \mid} M_{1} M_{2} M_{3}\left(\tilde{\Psi}_{P} \Gamma^{[P} \Gamma_{\mid N) M_{1} M_{2} M_{3}} \Gamma^{Q]} \Psi_{Q}\right) \\
& -\frac{1}{96} e^{\phi / 4} G_{M_{1} \ldots M_{4}}\left\{\left(\tilde{\Psi}_{P} \Gamma_{(M} \Gamma^{M_{1} \ldots M_{4}} \Gamma^{P} \Psi_{N)}\right)-\left(\tilde{\Psi}_{P} \Gamma^{P} \Gamma^{M_{1} \ldots M_{4}} \Gamma_{(M} \Psi_{N)}\right)\right. \\
& \left.+\frac{1}{2} g_{M N}\left(\tilde{\Psi}^{P} \Gamma_{[P} \Gamma^{M_{1} \ldots M_{4}} \Gamma_{Q]} \Psi^{Q}\right)\right\}-\frac{1}{8} g_{M N} L_{\Psi^{4}}+\frac{\delta L_{\Psi^{4}}}{\delta g^{M N}},
\end{aligned}
$$

where we have set: $\Phi_{M N}^{2}:=\Phi_{M M_{2} \ldots M_{p}} \Phi_{N} M_{2} \ldots M_{p}$, for any $p$-form $\Phi$. In the Einstein equation above we have not included the gravitino couplings to the two- and three-forms: these vanish in the ALE background, as we will see in the following. Moreover, we have refrained from spelling out explicitly the quartic gravitino terms, as they are numerous and not particularly enlightening. We will calculate them explicitly later on in the case of the ALE space in section 3.3. 
Form EOM's, ${ }^{8}$

$$
\begin{aligned}
0= & \mathrm{d} \star\left[e^{3 \phi / 2} F-\frac{1}{2} e^{3 \phi / 4}\left(\tilde{\Psi}^{M} \Gamma_{[M} \Gamma^{(2)} \Gamma_{N]} \Gamma_{11} \Psi^{N}\right)\right] \\
& \left.+H \wedge \star e^{\phi / 2} G+\frac{1}{2} e^{\phi / 4}\left(\tilde{\Psi}^{M} \Gamma_{[M} \Gamma^{(4)} \Gamma_{N]} \Psi^{N}\right)\right] \\
0= & \mathrm{d} \star\left[e^{-\phi} H-\frac{1}{2} e^{-\phi / 2}\left(\tilde{\Psi}^{M} \Gamma_{[M} \Gamma^{(3)} \Gamma_{N]} \Gamma_{11} \Psi^{N}\right)\right] \\
& +e^{\phi / 2} F_{\wedge} \star\left[e^{\phi / 2} G+\frac{1}{2} e^{\phi / 4}\left(\tilde{\Psi}^{M} \Gamma_{[M} \Gamma^{(4)} \Gamma_{N]} \Psi^{N}\right)\right] \\
& -\frac{1}{2} G \wedge G+m \star\left[e^{3 \phi / 2} F-\frac{1}{2} e^{3 \phi / 4}\left(\tilde{\Psi}^{M} \Gamma_{[M} \Gamma^{(2)} \Gamma_{N]} \Gamma_{11} \Psi^{N}\right)\right] \\
0= & \mathrm{d} \star\left[e^{\phi / 2} G+\frac{1}{2} e^{\phi / 4}\left(\tilde{\Psi}^{M} \Gamma_{[M} \Gamma^{(4)} \Gamma_{N]} \Psi^{N}\right)\right]-H \wedge G,
\end{aligned}
$$

where $\Gamma^{(p)}:=\frac{1}{p !} \Gamma_{M_{1} \ldots M_{p}} \mathrm{~d} x^{M_{p}} \wedge \cdots \wedge \mathrm{d} x^{M_{1}}$. In addition the forms obey the Bianchi identities,

$$
\mathrm{d} F=m H ; \quad \mathrm{d} H=0 ; \quad \mathrm{d} G=H \wedge F .
$$

\subsection{Consistent truncation without condensates}

The truncation of [20] contains the four real scalars $(A, \chi, \phi, \xi)$, with $A$ related to the volume modulus $v$ of section 2: it does not capture all the scalars of the universal sector of $\mathcal{N}=2$ supergravity, since it does not include the vectors and it truncates the two scalars $\xi^{\prime}, b$ of section 2. We must therefore expand the ansatz of [20] to include the "missing" fields, at the same time taking the limit to the massless IIA theory, $m \rightarrow 0$. Explicitly we set,

$F=\mathrm{d} \alpha ; \quad H=\mathrm{d} \chi_{\wedge} J+\mathrm{d} \beta ; \quad G=\varphi \operatorname{vol}_{4}+\frac{1}{2} c_{0} J_{\wedge} J+J_{\wedge}(\mathrm{d} \gamma-\alpha \wedge \mathrm{d} \chi)-\frac{1}{2} \mathrm{~d} \xi \wedge \operatorname{Im} \Omega-\frac{1}{2} \mathrm{~d} \xi^{\prime} \operatorname{Re} \Omega$,

where $c_{0}$ is a real constant and $\varphi(x)$ is a $4 \mathrm{~d}$ scalar. We have chosen to express $H$ in terms of the $4 \mathrm{~d}$ potential $\beta$ instead of the axion. Taking into account that for a CY we have $\mathrm{d} J=\mathrm{d} \Omega=0$, this ansatz can be seen to automatically satisfy the Bianchi identities (3.6) in the massless limit. Our ansatz for the ten-dimensional metric reads,

$$
\mathrm{d} s_{(10)}^{2}=e^{2 A(x)}\left(e^{2 B(x)} g_{\mu \nu} \mathrm{d} x^{\mu} \mathrm{d} x^{\nu}+g_{m n} \mathrm{~d} y^{m} \mathrm{~d} y^{n}\right)
$$

${ }^{8}$ We are using "superspace conventions" as in [48] so that,

$$
\Phi_{(p)}=\frac{1}{p !} \Phi_{m_{1} \ldots m_{p}} \mathrm{~d} x^{m_{p}} \wedge \ldots \wedge \mathrm{d} x^{m_{1}} ; \quad \mathrm{d}\left(\Phi_{(p) \wedge} \Psi_{(q)}\right)=\Phi_{(p) \wedge} \mathrm{d} \Psi_{(q)}+(-1)^{q} \mathrm{~d} \Phi_{(p) \wedge} \wedge \Psi_{(q)} .
$$

In $D$ dimensions the Hodge star is defined as follows,

$$
\star\left(\mathrm{d} x^{a_{1}} \wedge \cdots \wedge \mathrm{d} x^{a_{p}}\right)=\frac{1}{(D-p) !} \varepsilon_{b_{1} \ldots b_{10-p}}^{a_{1} \ldots a_{p}} x^{b_{1}} \wedge \cdots \wedge \mathrm{d} x^{b_{10-p}} .
$$


where the scalars $A, B$ only depend on the four-dimensional coordinates $x^{\mu}$. This gives,

$$
\begin{aligned}
F_{\mu \nu}^{2} & =e^{-2 A-2 B} \mathrm{~d} \alpha_{\mu \nu}^{2} ; \quad F^{2}=e^{-4 A-4 B} \mathrm{~d} \alpha^{2} \\
H_{m n}^{2} & =2 e^{-4 A-2 B}(\partial \chi)^{2} g_{m n} ; \quad H_{\mu \nu}^{2}=6 e^{-4 A} \partial_{\mu} \chi \partial_{\nu} \chi+e^{-4 A-4 B} h_{\mu \nu}^{2} \\
H^{2} & =18 e^{-6 A-2 B}(\partial \chi)^{2}+e^{-6 A-6 B} h^{2} \\
G_{m n}^{2} & =3 e^{-6 A-2 B}\left[(\partial \xi)^{2}+\left(\partial \xi^{\prime}\right)^{2}\right] g_{m n}+12 e^{-6 A} c_{0}^{2} g_{m n}+3 e^{-6 A-4 B}(\mathrm{~d} \gamma-\alpha \wedge \mathrm{d} \chi)^{2} g_{m n} \\
G_{\mu \nu}^{2} & =-6 e^{-6 A-6 B} \varphi^{2} g_{\mu \nu}+6 e^{-6 A}\left(\partial_{\mu} \xi \partial_{\nu} \xi+\partial_{\mu} \xi^{\prime} \partial_{\nu} \xi^{\prime}\right)+18 e^{-6 A-2 B}(\mathrm{~d} \gamma-\alpha \wedge \mathrm{d} \chi)_{\mu \nu}^{2} \\
G^{2} & =-24 e^{-8 A-8 B} \varphi^{2}+24 e^{-8 A-2 B}\left[(\partial \xi)^{2}+\left(\partial \xi^{\prime}\right)^{2}\right]+72 c_{0}^{2} e^{-8 A}+36 e^{-8 A-4 B}(\mathrm{~d} \gamma-\alpha \wedge \mathrm{d} \chi)^{2},
\end{aligned}
$$

where the contractions on the left-hand sides above are computed with respect to the tendimensional metric; the contractions on the right-hand sides are taken with respect to the unwarped metric. It is also useful to note the following expressions,

$$
\begin{aligned}
\star_{10} F= & \frac{1}{6} e^{6 A} \star_{4} \mathrm{~d} \alpha_{\wedge} J^{3} \\
\star_{10} H= & \frac{1}{2} e^{4 A+2 B} \star_{4} \mathrm{~d} \chi_{\wedge} J^{2}+\frac{1}{6} e^{4 A-2 B} \star_{4} h_{\wedge} J^{3} \\
\star_{10} G= & -\frac{1}{6} \varphi e^{2 A-4 B} J^{3}+c_{0} e^{2 A+4 B} \operatorname{vol}_{4}{ }^{\wedge} J+\frac{1}{2} e^{2 A} \star_{4}(\mathrm{~d} \gamma-\alpha \wedge \mathrm{d} \chi) \wedge J^{2} \\
& +\frac{1}{2} e^{2 A+2 B} \star_{4} \mathrm{~d} \xi \wedge \operatorname{Re} \Omega-\frac{1}{2} e^{2 A+2 B} \star_{4} \mathrm{~d} \xi^{\prime}{ }_{\wedge} \operatorname{Im} \Omega,
\end{aligned}
$$

where the four-dimensional Hodge-star is taken with respect to the unwarped metric.

Plugging the above ansatz into the ten-dimensional EOM (3.3)-(3.5) we obtain the following: the internal $(m, n)$-components of the Einstein equations read,

$$
\begin{aligned}
0= & e^{-8 A-2 B} \nabla^{\mu}\left(e^{8 A+2 B} \partial_{\mu} A\right)-\frac{1}{32} e^{3 \phi / 2-2 A-2 B} \mathrm{~d} \alpha^{2}+\frac{1}{8} e^{-\phi-4 A}(\partial \chi)^{2}-\frac{1}{48} e^{-\phi-4 A-4 B} h^{2} \\
& -\frac{1}{32} e^{\phi / 2-6 A-2 B}(\mathrm{~d} \gamma-\alpha \wedge \mathrm{d} \chi)^{2}+\frac{1}{16} e^{\phi / 2-6 A}\left[(\partial \xi)^{2}+\left(\partial \xi^{\prime}\right)^{2}\right] \\
& +\frac{3}{16} e^{\phi / 2-6 A-6 B} \varphi^{2}+\frac{7}{16} e^{\phi / 2-6 A+2 B} c_{0}^{2} .
\end{aligned}
$$

The external $(\mu, \nu)$-components read,

$$
\begin{aligned}
R_{\mu \nu}^{(4)}= & g_{\mu \nu}\left(\nabla^{2} A+\nabla^{2} B+8(\partial A)^{2}+2(\partial B)^{2}+10 \partial A \cdot \partial B\right) \\
& -8 \partial_{\mu} A \partial_{\nu} A-2 \partial_{\mu} B \partial_{\nu} B-16 \partial_{(\mu} A \partial_{\nu)} B+8 \nabla_{\mu} \partial_{\nu} A+2 \nabla_{\mu} \partial_{\nu} B \\
& +\frac{3}{2} e^{-\phi-4 A} \partial_{\mu} \chi \partial_{\nu} \chi+\frac{1}{2} e^{3 \phi / 2-2 A-2 B} \mathrm{~d} \alpha_{\mu \nu}^{2}+\frac{1}{4} e^{\phi-4 A-4 B} h_{\mu \nu}^{2}+\frac{1}{2} \partial_{\mu} \phi \partial_{\nu} \phi \\
& +\frac{1}{2} e^{\phi / 2-6 A}\left(\partial_{\mu} \xi \partial_{\nu} \xi+\partial_{\mu} \xi^{\prime} \partial_{\nu} \xi^{\prime}\right)+\frac{3}{2} e^{\phi / 2-6 A-2 B}(\mathrm{~d} \gamma-\alpha \wedge \mathrm{d} \chi)_{\mu \nu}^{2} \\
& +\frac{1}{16} g_{\mu \nu}\left(-\frac{1}{2} e^{3 \phi / 2-2 A-2 B} \mathrm{~d} \alpha^{2}-\frac{1}{3} e^{\phi-4 A-4 B} h^{2}-3 e^{\phi / 2-6 A}\left[(\partial \xi)^{2}+\left(\partial \xi^{\prime}\right)^{2}\right]\right. \\
& \left.-6 e^{-\phi-4 A}(\partial \chi)^{2}-5 e^{\phi / 2-6 A-6 B} \varphi^{2}-9 c_{0}^{2} e^{\phi / 2-6 A+2 B}-\frac{9}{2} e^{\phi / 2-6 A-2 B}(\mathrm{~d} \gamma-\alpha \wedge \mathrm{d} \chi)^{2}\right),
\end{aligned}
$$


while the mixed $(\mu, m)$-components are automatically satisfied. The dilaton equation reads,

$$
\begin{aligned}
0= & e^{-10 A-4 B} \nabla^{\mu}\left(e^{8 A+2 B} \partial_{\mu} \phi\right)-\frac{1}{4} e^{\phi / 2-8 A-2 B}\left[(\partial \xi)^{2}+\left(\partial \xi^{\prime}\right)^{2}\right]-\frac{3}{8} e^{3 \phi / 2-4 A-4 B} \mathrm{~d} \alpha^{2} \\
& +\frac{3}{2} e^{-\phi-6 A-2 B}(\partial \chi)^{2}+\frac{1}{12} e^{-\phi-6 A-6 B} h^{2} \\
& +\frac{1}{4} e^{\phi / 2-8 A-8 B} \varphi^{2}-\frac{3}{4} c_{0}^{2} e^{\phi / 2-8 A}-\frac{3}{8} e^{\phi / 2-8 A-4 B}(\mathrm{~d} \gamma-\alpha \wedge \mathrm{d} \chi)^{2}
\end{aligned}
$$

The $F$-form equation of motion reduces to the condition,

$$
\mathrm{d}\left(e^{3 \phi / 2+6 A} \star_{4} \mathrm{~d} \alpha\right)=\varphi e^{\phi / 2+2 A-4 B} \mathrm{~d} \beta-3 e^{\phi / 2+2 A} \mathrm{~d} \chi \wedge \star_{4}(\mathrm{~d} \gamma-\alpha \wedge \mathrm{d} \chi) .
$$

The $H$-form equation reduces to the following two equations,

$$
\begin{aligned}
\mathrm{d}\left(e^{-\phi+4 A+2 B} \star_{4} \mathrm{~d} \chi\right)= & c_{0} \varphi \operatorname{vol}_{4}+(\mathrm{d} \gamma-\alpha \wedge \mathrm{d} \chi) \wedge(\mathrm{d} \gamma-\alpha \wedge \mathrm{d} \chi) \\
& -e^{\phi / 2+2 A} \mathrm{~d} \alpha \wedge \star_{4}(\mathrm{~d} \gamma-\alpha \wedge \mathrm{d} \chi)
\end{aligned}
$$

and,

$$
\mathrm{d}\left(e^{-\phi+4 A-2 B} \star_{4} \mathrm{~d} \beta\right)=3 c_{0}(\mathrm{~d} \gamma-\alpha \wedge \mathrm{d} \chi)-\mathrm{d} \xi \wedge \mathrm{d} \xi^{\prime}+e^{\phi / 2+2 A-4 B} \varphi \mathrm{d} \alpha .
$$

The $G$-form equation of motion reduces to,

$$
\begin{aligned}
\mathrm{d}\left(e^{\phi / 2+2 A+2 B} \star_{4} \mathrm{~d} \xi\right) & =h_{\wedge} \mathrm{d} \xi^{\prime} \\
\mathrm{d}\left(e^{\phi / 2+2 A+2 B} \star_{4} \mathrm{~d} \xi^{\prime}\right) & =-h_{\wedge} \mathrm{d} \xi \\
\mathrm{d}\left(e^{\phi / 2+2 A} \star_{4}(\mathrm{~d} \gamma-\alpha \wedge \mathrm{d} \chi)\right) & =2 \mathrm{~d} \chi_{\wedge} \mathrm{d} \gamma+c_{0} \mathrm{~d} \beta
\end{aligned}
$$

together with the constraint,

$$
0=\mathrm{d}\left(\varphi e^{\phi / 2+2 A-4 B}+3 c_{0} \chi\right)
$$

This can be readily integrated to give,

$$
\varphi=e^{-\phi / 2-18 A}\left(c_{1}-3 c_{0} \chi\right) .
$$

Since $\chi$ only appears in the equations of motion through its derivatives or through $\varphi$, we may absorb $c_{1}$ by redefining $\chi$. This corresponds to a gauge transformation of the ten-dimensional $B$-field. We will thus set $c_{1}$ to zero in the following.

The Lagrangian. As we can see from (3.8) the scalar $B(x)$ can be redefined away by absorbing it in the $4 \mathrm{~d}$ metric. This freedom can be exploited in order to obtain a $4 \mathrm{~d}$ consistent truncation directly in the Einstein frame. The appropriate choice is,

$$
B=-4 A
$$


With this choice one can check that the ten-dimensional equations given in (3.11)-(3.18) all follow from the $4 \mathrm{~d}$ action,

$$
\begin{aligned}
S_{4}=\int \mathrm{d}^{4} x \sqrt{g}( & R-24(\partial A)^{2}-\frac{1}{2}(\partial \phi)^{2}-\frac{3}{2} e^{-4 A-\phi}(\partial \chi)^{2}-\frac{1}{2} e^{-6 A+\phi / 2}\left[(\partial \xi)^{2}+\left(\partial \xi^{\prime}\right)^{2}\right] \\
& -\frac{1}{4} e^{3 \phi / 2+6 A} \mathrm{~d} \alpha^{2}-\frac{3}{4} e^{\phi / 2+2 A}(\mathrm{~d} \gamma-\alpha \wedge \mathrm{d} \chi)^{2}-\frac{1}{12} e^{-\phi+12 A} \mathrm{~d} \beta^{2} \\
& \left.-\frac{9}{2} e^{-\phi / 2-18 A} c_{0}^{2} \chi^{2}-\frac{3}{2} e^{\phi / 2-14 A} c_{0}^{2}\right) \\
& +\int 3 c_{0} \mathrm{~d} \gamma_{\wedge} \beta+3 c_{0} \chi \alpha \wedge \mathrm{d} \beta+3 \chi \mathrm{d} \gamma_{\wedge} \mathrm{d} \gamma-\beta \wedge \mathrm{d} \xi \wedge \mathrm{d} \xi^{\prime}
\end{aligned}
$$

Furthermore equation (3.16) can be solved in order to express $\mathrm{d} \beta$ in terms of a scalar $b$ (the "axion"),

$$
\mathrm{d} \beta=e^{\phi-12 A} \star_{4}\left[\mathrm{~d} b+\frac{1}{2}\left(\xi \mathrm{d} \xi^{\prime}-\xi^{\prime} \mathrm{d} \xi\right)+3 c_{0}(\gamma-\chi \alpha)\right],
$$

where we chose the gauge most symmetric in $\xi, \xi^{\prime}$. The Lagrangian becomes, in terms of the axion,

$$
\begin{aligned}
S_{4}=\int \mathrm{d}^{4} x \sqrt{g}( & R-24(\partial A)^{2}-\frac{1}{2}(\partial \phi)^{2}-\frac{3}{2} e^{-4 A-\phi}(\partial \chi)^{2}-\frac{1}{2} e^{-6 A+\phi / 2}\left[(\partial \xi)^{2}+\left(\partial \xi^{\prime}\right)^{2}\right] \\
& -\frac{1}{4} e^{3 \phi / 2+6 A} \mathrm{~d} \alpha^{2}-\frac{3}{4} e^{\phi / 2+2 A}(\mathrm{~d} \gamma-\alpha \wedge \mathrm{d} \chi)^{2}-\frac{1}{2} e^{\phi-12 A}(\mathrm{~d} b+\omega)^{2} \\
& \left.-\frac{9}{2} e^{-\phi / 2-18 A} c_{0}^{2} \chi^{2}-\frac{3}{2} e^{\phi / 2-14 A} c_{0}^{2}\right)+\int 3 \chi \mathrm{d} \gamma \wedge \mathrm{d} \gamma
\end{aligned}
$$

where we have set,

$$
\omega:=\frac{1}{2}\left(\xi \mathrm{d} \xi^{\prime}-\xi^{\prime} \mathrm{d} \xi\right)+3 c_{0}(\gamma-\chi \alpha) .
$$

Including background three-form flux. We can include background three-form flux by modifying the form ansatz (3.7) as follows,

$$
\begin{aligned}
& F=\mathrm{d} \alpha ; \quad H=\mathrm{d} \chi_{\wedge} J+\mathrm{d} \beta+\frac{1}{2} \operatorname{Re}\left(b_{0} \Omega^{*}\right) \\
& G=\varphi \operatorname{vol}_{4}+\frac{1}{2} c_{0} J_{\wedge} J+J_{\wedge}(\mathrm{d} \gamma-\alpha \wedge \mathrm{d} \chi)-\frac{1}{2} D \xi_{\wedge} \operatorname{Im} \Omega-\frac{1}{2} D \xi^{\prime} \wedge \operatorname{Re} \Omega,
\end{aligned}
$$

where we have introduced a background charge $b_{0} \in \mathbb{C}$. The covariant derivatives are given by,

$$
D \xi:=\mathrm{d} \xi+b_{1} \alpha ; \quad D \xi^{\prime}:=\mathrm{d} \xi^{\prime}+b_{2} \alpha,
$$

where we set $b_{0}=i b_{1}+b_{2}$. We see that the inclusion of a background charge for the three-form has the effect of gauging the isometries of the RR axions.

The modified form ansatz (3.25) is such that it automatically satisfies the Bianchi identities. At the level of the Lagrangian, the modification amounts to replacing: $\mathrm{d} \xi \rightarrow D \xi$, $\mathrm{d} \xi^{\prime} \rightarrow D \xi^{\prime}$, and adding a potential term,

$$
S_{4} \rightarrow S_{4}-\int \mathrm{d}^{4} x \sqrt{g} \frac{1}{2}\left|b_{0}\right|^{2} e^{-\phi-12 A} .
$$




\subsection{Consistent truncation with condensates}

In Euclidean signature the supersymmetric IIA action is constructed via the procedure of holomorphic complexification, see e.g. [49]. This amounts to first expressing the Lorentzian action in terms of $\tilde{\Psi}_{M}$ instead of $\bar{\Psi}_{M}$ (which makes no difference in Lorentzian signature) and then Wick-rotating, see appendix A for our spinor and gamma-matrix conventions. In this way one obtains a (complexified) Euclidean action which is formally identical to the Lorentzian one, with the difference that now the two chiralities $\Psi_{M}^{ \pm}$, should be thought of as independent complex spinors (there are no Majorana Weyl spinors in ten Euclidean dimensions). Although the gravitino $\Psi_{M}$ is complex in Euclidean signature, its complex conjugate does not appear in the action, hence the term "holomorphic complexification".

Since we are interested in the case where only the $4 \mathrm{~d}$ gravitino condenses, we expand the $10 \mathrm{~d}$ gravitino as follows,

$$
\Psi_{m}=0 ; \quad \Psi_{\mu+}=\psi_{\mu+} \otimes \eta-\psi_{\mu-} \otimes \eta^{c} ; \quad \Psi_{\mu-}=\psi_{\mu+}^{\prime} \otimes \eta^{c}-\psi_{\mu-}^{\prime} \otimes \eta,
$$

so that,

$$
\tilde{\Psi}_{\mu+}=\tilde{\psi}_{\mu+} \otimes \tilde{\eta}+\tilde{\psi}_{\mu-} \otimes \tilde{\eta}^{c} ; \quad \tilde{\Psi}_{\mu-}=\tilde{\psi}_{\mu+}^{\prime} \otimes \tilde{\eta}^{c}+\tilde{\psi}_{\mu-}^{\prime} \otimes \tilde{\eta} .
$$

In Lorentzian signature the positive- and negative-chirality $4 \mathrm{~d}$ vector-spinors above are related though complex conjugation: $\bar{\theta}_{+}^{\mu}=\tilde{\theta}_{-}^{\mu}, \bar{\theta}_{-}^{\mu}=-\tilde{\theta}_{+}^{\mu}$, so that $\Psi_{M}$ is Majorana in 10d: $\bar{\Psi}_{M}=\tilde{\Psi}_{M}$. Upon Wick-rotating to Euclidean signature this is no longer true, and the two chiralities transform in independent representations. As already mentioned, in the present paper we focus on the contribution of ALE gravitational instantons to the fermion condensate. In this case there are no negative-chirality zeromodes and we can set,

$$
\psi_{-}^{\mu}=\psi_{-}^{\prime \mu}=0 .
$$

For any two $4 \mathrm{~d}$ positive-chirality vector-spinors, $\theta_{+}^{\mu}, \chi_{+}^{\mu}$, the only nonvanishing bilinears read,

$$
\left(\theta_{+}^{\left[\mu_{1}\right.} \gamma^{\mu_{2} \mu_{3}} \chi_{+}^{\left.\mu_{4}\right]}\right)=\frac{i^{s}}{12} \varepsilon^{\mu_{1} \mu_{2} \mu_{3} \mu_{4}}\left(\theta_{+}^{\lambda} \gamma_{\lambda \rho} \chi_{+}^{\rho}\right) ; \quad\left(\theta_{+}^{\lambda} \chi_{\lambda+}\right),
$$

where we used the Fierz identity (A.4) and the Hodge duality relations (A.5); $s=1,2$ for Lorentzian, Euclidean signature respectively. Ultimately we will be interested in gammatraceless vector-spinors,

$$
\gamma_{\mu} \theta_{+}^{\mu}=\gamma_{\mu} \chi_{+}^{\mu}=0
$$

since all ALE zeromodes can be put in this gauge [29]. In this case we obtain the additional relation,

$$
\left(\theta_{+}^{\lambda} \gamma_{\lambda \rho} \chi_{+}^{\rho}\right)=-\left(\theta_{+}^{\lambda} \chi_{\lambda+}\right)
$$

Assuming, as is the case for ALE spaces, that only positive-chirality zeromodes exist in four dimensions, cf. (3.30), the only nonvanishing bilinear condensates that appear in the equations of motion are proportional to,

$$
\mathcal{A}:=\left(\tilde{\psi}_{\mu+} \gamma^{\mu \nu} \psi_{\nu+}^{\prime}\right)=-\left(\tilde{\psi}_{+}^{\mu} \psi_{\mu+}^{\prime}\right),
$$

where in the second equality we have assumed that $\psi_{+}^{\mu}, \psi_{+}^{\prime \mu}$ are gamma-traceless, cf. (3.32). 
Furthermore we note the following useful results,

$$
\begin{aligned}
\left(\tilde{\Psi}_{\rho} \Gamma_{(\mu} \Gamma^{M_{1} \ldots M_{4}} \Gamma^{\rho} \Psi_{\nu)}\right) G_{M_{1} \ldots M_{4}} & =24\left(3 c_{0} e^{-4 A}+\varphi e^{-4 A-4 B}\right) \mathcal{A} g_{\mu \nu} \\
\left(\tilde{\Psi}_{\rho} \Gamma_{\sigma} \Gamma_{(\mu}{ }^{M_{2} M_{3} M_{4}} \Gamma^{\rho} \Psi^{\sigma}\right) G_{\nu) M_{2} M_{3} M_{4}} & =24 \varphi e^{-4 A-4 B} \mathcal{A} g_{\mu \nu} \\
\left(\tilde{\Psi}_{\rho} \Gamma_{\sigma} \Gamma_{(m}{ }^{M_{2} M_{3} M_{4}} \Gamma^{\rho} \Psi^{\sigma}\right) G_{n) M_{2} M_{3} M_{4}} & =48 c_{0} \mathcal{A} e^{-4 A-2 B} g_{m n}
\end{aligned}
$$

where on the left-hand sides above we used the warped metric for the contractions, while on the right-hand sides we used the unwarped metric. In the $4 \mathrm{~d}$ theory, these bilinears receive contributions from the $\mathrm{EH}$ instanton at one loop in the gravitational coupling.

In the presence of gravitino condensates the equations of motion (3.11)-(3.18) are modified as follows: the internal $(m, n)$-components of the Einstein equations read,

$$
0=e^{-8 A-2 B} \nabla^{\mu}\left(e^{8 A+2 B} \partial_{\mu} A\right)+\cdots+\frac{1}{4}\left(\varphi e^{\phi / 4-4 A-4 B}-c_{0} e^{\phi / 4-4 A}\right) \mathcal{A}-\frac{1}{8} e^{2 A+2 B} L_{\Psi^{4}},
$$

where the ellipses stand for terms that are identical to the case without fermion condensates. The external $(\mu, \nu)$-components read,

$$
R_{\mu \nu}^{(4)}=\cdots-\frac{1}{2} g_{\mu \nu} e^{\phi / 4-4 A-4 B} \varphi \mathcal{A}
$$

while the mixed $(\mu, m)$-components are automatically satisfied. The dilaton equation reads,

$$
0=e^{-10 A-4 B} \nabla^{\mu}\left(e^{8 A+2 B} \partial_{\mu} \phi\right)+\cdots+\frac{1}{4}\left(3 c_{0} e^{\phi / 4+2 A}+\varphi e^{\phi / 4+2 A-4 B}\right) \mathcal{A} .
$$

The $F$-form and $H$-form equations are modified as follows,

$$
\mathrm{d}\left(e^{3 \phi / 2+6 A} \star_{4} \mathrm{~d} \alpha\right)=\ldots+e^{\phi / 4+4 A-2 B} \mathcal{A} \mathrm{d} \beta
$$

and,

$$
\mathrm{d}\left(e^{-\phi+4 A-2 B} \star_{4} \mathrm{~d} \beta\right)=\ldots+e^{\phi / 4+4 A-2 B} \mathcal{A} \mathrm{d} \alpha,
$$

respecively. The $G$-form equation of motion remains unchanged except for the constraint,

$$
0=\mathrm{d}\left(\varphi e^{\phi / 2+2 A-4 B}+3 c_{0} \chi+e^{\phi / 4+4 A-2 B} \mathcal{A}\right) .
$$

In deriving the above we have taken into account that,

$$
\left(\tilde{\Psi}^{M} \Gamma_{[M} \Gamma^{(4)} \Gamma_{N]} \Psi^{N}\right)=2 \mathcal{A} e^{2 A+2 B}\left(\operatorname{vol}_{4}-\frac{1}{2} e^{-4 B} J_{\wedge} J\right)
$$

At this stage it is important to notice that the new $\mathcal{A}$ terms in the flux equations (3.39) and (3.40) exactly compensate the modification of $\varphi$ in (3.41), so that the form equations are ultimately unchanged in the presence of fermion condensates. 
Of the 24 quartic gravitino terms that appear in the action of [42] only the following are nonvanishing,

$$
\begin{aligned}
\left(\tilde{\Psi}_{\mu} \Gamma_{11} \Psi_{\nu}\right)\left(\tilde{\Psi}^{\mu} \Gamma_{11} \Psi^{\nu}\right) & =4\left(\tilde{\psi}_{+}^{[\mu} \psi_{+}^{\prime \nu]}\right)^{2} e^{-4 A-4 B} \\
\left(\tilde{\Psi}^{\mu_{1}} \Gamma_{11} \Gamma_{\mu_{1} \ldots \mu_{4}} \Psi^{\mu_{2}}\right)\left(\tilde{\Psi}^{\mu_{3}} \Gamma_{11} \Psi^{\mu_{4}}\right) & =-\frac{1}{6}\left(\tilde{\Psi}^{\mu_{1}} \Gamma_{\mu_{1} \ldots \mu_{4} m n} \Psi^{\mu_{2}}\right)\left(\tilde{\Psi}^{\mu_{3}} \Gamma^{m n} \Psi^{\mu_{4}}\right) \\
& =-\left(8 \tilde{\psi}_{[\nu+} \psi_{\rho]+}^{\prime}+4 \tilde{\psi}_{+}^{\mu} \gamma_{\rho \nu} \psi_{\mu+}^{\prime}\right)\left(\tilde{\psi}_{+}^{\rho} \psi_{+}^{\prime \nu}\right) e^{-4 A-4 B} \\
\left(\tilde{\Psi}^{\left[M_{1}\right.} \Gamma^{M_{2} M_{3}} \Psi^{\left.M_{4}\right]}\right)^{2} & =4\left(\tilde{\psi}_{+}^{\left[\mu_{1}\right.} \gamma^{\mu_{2} \mu_{3}} \psi_{+}^{\left.\prime \mu_{4}\right]}\right)^{2} e^{-4 A-4 B}-\frac{2}{3}\left(\tilde{\psi}_{+}^{[\mu} \psi_{+}^{\prime \nu]}\right)^{2} e^{-4 A-4 B},
\end{aligned}
$$

where for the contractions on the left-, right-hand sides above we have used the warped, unwarped metric respectively. We thus obtain, cf. (3.1),

$$
\begin{aligned}
L_{\Psi^{4}}= & \frac{1}{4}\left(\tilde{\Psi}_{M} \Gamma_{11} \Psi_{N}\right)^{2}+\frac{1}{8} \tilde{\Psi}^{M_{1}} \Gamma_{11} \Gamma_{M_{1} \cdots M_{4}} \Psi^{M_{2}} \tilde{\Psi}^{M_{3}} \Gamma_{11} \Psi^{M_{4}} \\
& +\frac{1}{16} \tilde{\Psi}^{M_{1}} \Gamma_{M_{1} \cdots M_{6}} \Psi^{M_{2}} \tilde{\Psi}^{M_{3}} \Gamma^{M_{4} M_{5}} \Psi^{M_{6}}+\frac{3}{4}\left(\tilde{\Psi}_{\left[M_{1}\right.} \Gamma_{M_{2} M_{3}} \Psi_{\left.M_{4}\right]}\right)^{2} \\
= & e^{-4 A-4 B} \mathcal{B},
\end{aligned}
$$

where we have defined,

$$
\mathcal{B}:=-\frac{3}{2}\left(\tilde{\psi}_{[\mu} \psi_{\nu]}^{\prime}\right)^{2}+\left(\tilde{\psi}^{\mu} \gamma_{\rho \nu} \psi_{\mu}^{\prime}\right)\left(\tilde{\psi}^{\rho} \psi^{\prime \nu}\right)+3\left(\tilde{\psi}_{\left[\mu_{1}\right.} \gamma_{\mu_{2} \mu_{3}} \psi_{\left.\mu_{4}\right]}^{\prime}\right)^{2},
$$

which does not depend on the warp factor. In the $4 \mathrm{~d}$ theory, at one-loop order in the gravitational coupling, the quartic gravitino term receives contributions from the ALE instanton with $\tau=2$ (four spin-3/2 zeromodes).

The Lagrangian. Imposing (3.20) as before, and solving once again for $\varphi$,

$$
\varphi=e^{-\phi / 2-18 A}\left(-3 c_{0} \chi-e^{\phi / 4+12 A} \mathcal{A}\right),
$$

it can now be seen that the ten-dimensional equations in the presence of gravitino condensates all follow from the $4 \mathrm{~d}$ action,

$$
\begin{aligned}
S_{4}= & \int \mathrm{d}^{4} x \sqrt{g}\left(R-24(\partial A)^{2}-\frac{1}{2}(\partial \phi)^{2}-\frac{3}{2} e^{-4 A-\phi}(\partial \chi)^{2}-\frac{1}{2} e^{-6 A+\phi / 2}\left[(D \xi)^{2}+\left(D \xi^{\prime}\right)^{2}\right]\right. \\
& \left.-\frac{1}{4} e^{3 \phi / 2+6 A} \mathrm{~d} \alpha^{2}-\frac{3}{4} e^{\phi / 2+2 A}(\mathrm{~d} \gamma-\alpha \wedge \mathrm{d} \chi)^{2}-\frac{1}{12} e^{-\phi+12 A} \mathrm{~d} \beta^{2}-V\right) \\
& +\int 3 c_{0} \mathrm{~d} \gamma_{\wedge} \beta+3 c_{0} \chi \alpha \wedge \mathrm{d} \beta+3 \chi \mathrm{d} \gamma_{\wedge} \mathrm{d} \gamma-\beta_{\wedge} D \xi_{\wedge} D \xi^{\prime}
\end{aligned}
$$

where the potential of the theory is given by,

$$
\begin{aligned}
V(\chi, \phi, A)= & \frac{9}{2} c_{0}^{2} \chi^{2} e^{-\phi / 2-18 A}+\frac{3}{2} c_{0}^{2} e^{\phi / 2-14 A}+\frac{1}{2}\left|b_{0}\right|^{2} e^{-\phi-12 A} \\
& +3 c_{0} \chi \mathcal{A} e^{-\phi / 4-6 A}-3 c_{0} \mathcal{A} e^{\phi / 4-4 A}+e^{6 A}\left(\mathcal{B}+\frac{1}{2} \mathcal{A}^{2}\right) .
\end{aligned}
$$


Note that in integrating the $4 \mathrm{~d}$ Einstein equation (3.37), care must be taken to first substitute in the right-hand side the value of $\varphi$ from (3.46), and take into account the variation of the condensates $\mathcal{A}, \mathcal{B}$ with respect to the metric.

The relation (3.22) between $\beta$ and the axion is unchanged. In terms of the axion, the action reads,

$$
\begin{aligned}
S_{4}=\int \mathrm{d}^{4} x \sqrt{g}( & R-24(\partial A)^{2}-\frac{1}{2}(\partial \phi)^{2}-\frac{3}{2} e^{-4 A-\phi}(\partial \chi)^{2}-\frac{1}{2} e^{-6 A+\phi / 2}\left[(D \xi)^{2}+\left(D \xi^{\prime}\right)^{2}\right] \\
& -\frac{1}{4} e^{3 \phi / 2+6 A} \mathrm{~d} \alpha^{2}-\frac{3}{4} e^{\phi / 2+2 A}(\mathrm{~d} \gamma-\alpha \wedge \mathrm{d} \chi)^{2} \\
& \left.-\frac{1}{2} e^{\phi-12 A}(\mathrm{~d} b+\tilde{\omega})^{2}-V\right)+\int 3 \chi \mathrm{d} \gamma \wedge \mathrm{d} \gamma
\end{aligned}
$$

where we have set,

$$
\tilde{\omega}:=\frac{1}{2}\left(\xi D \xi^{\prime}-\xi^{\prime} D \xi\right)+3 c_{0}(\gamma-\chi \alpha) .
$$

Note that the three axionic scalars $\xi, \xi^{\prime}, b$, remain flat directions even in the presence of the flux and the condensate.

\subsection{Vacua}

Maximally-symmetric solutions of the effective $4 \mathrm{~d}$ theory (3.49) can be obtained by setting the vectors to zero,

$$
\alpha=\gamma=0,
$$

and minimizing the potential of the theory,

$$
\partial_{\chi} V\left(\chi_{0}, \phi_{0}, A_{0}\right)=\partial_{\phi} V\left(\chi_{0}, \phi_{0}, A_{0}\right)=\partial_{A} V\left(\chi_{0}, \phi_{0}, A_{0}\right)=0,
$$

where $(\chi, \phi, A)=\left(\chi_{0}, \phi_{0}, A_{0}\right)$ is the location of the minimum in field space. Then the Einstein equations determine the scalar curvature of the $4 \mathrm{~d}$ spacetime to be, ${ }^{9}$

$$
R=9 c_{0}^{2} \chi_{0}^{2} e^{-\phi_{0} / 2-18 A_{0}}+3 c_{0}^{2} e^{\phi_{0} / 2-14 A_{0}}+\left|b_{0}\right|^{2} e^{-\phi_{0}-12 A_{0}}+3 c_{0} \chi_{0} \mathcal{A} e^{-\phi_{0} / 4-6 A_{0}}-3 c_{0} \mathcal{A} e^{\phi_{0} / 4-4 A_{0}},
$$

and we assume that a Wick rotation has been performed back to Minkowski signature.

Condition (3.52) admits two classes of solutions.

Case 1: $c_{0}=0$. In this case, imposing the vanishing of $\partial_{\phi} V$ sets $b_{0}=0$, and the potential only depends on the warp factor $A$. A minimum is obtained at finite value of $A$ provided,

$$
\mathcal{B}=-\frac{1}{2} \mathcal{A}^{2}
$$

and requires the quartic condensate to be negative. From (3.53) it then follows that $R=0$, and we obtain a Minkowski $4 \mathrm{~d}$ vacuum. In fact the potential vanishes identically.

\footnotetext{
${ }^{9}$ Note that (3.53) is different from the standard relation $R=2 V_{0}$. This is because the condensates $\mathcal{A}, \mathcal{B}$ have non-trivial variations with respect to the metric.
} 
Case 2: $\boldsymbol{c}_{\mathbf{0}} \neq \mathbf{0}$. In this case (3.52) can be solved for finite values of $\phi$ and $A$. The value of $\chi$ at the minimum is given by,

$$
\chi_{0}=-\frac{\mathcal{A}}{3 c_{0}} g_{s}^{1 / 4} e^{12 A_{0}},
$$

where we have set $g_{s}:=e^{\phi_{0}}$. The values of $\phi_{0}$ and $A_{0}$ at the minimum can be adjusted arbitrarily, and determine $\left|b_{0}\right|$ and $c_{0}$ in terms of the condensates,

$$
\begin{aligned}
\left|b_{0}\right|^{2} & =\frac{3}{400} g_{s} e^{18 A_{0}}\left(40 \mathcal{B}-21 \mathcal{A}^{2} \mp 3 \mathcal{A} \sqrt{49 \mathcal{A}^{2}+80 \mathcal{B}}\right) \\
c_{0} & =\frac{1}{20} g_{s}^{-1 / 4} e^{10 A_{0}}\left(7 \mathcal{A} \pm \sqrt{49 \mathcal{A}^{2}+80 \mathcal{B}}\right)
\end{aligned}
$$

where the signs in $b_{0}$ and $c_{0}$ are correlated. Henceforth we will set $e^{A_{0}}=1$, since the warp factor at the minimum can be absorbed in $l_{Y}$.

Consistency of (3.56) requires the quartic condensate to obey the constraint,

$$
\mathcal{B}>0
$$

and correlates the sign of $\mathcal{A}$ with the two branches of the solution: the upper/lower sign in (3.56) corresponds to $\mathcal{A}$ negative/positive, respectively. ${ }^{10}$

From (3.53) it then follows that,

$$
R_{\mathrm{dS}}=3 g_{s}^{-1}\left|b_{0}\right|^{2} \propto l_{s}^{-2} e^{-2 c\left(l_{Y} / l_{s}\right)^{2}},
$$

up to a proportionality constant of order one. We thus obtain a de Sitter $4 \mathrm{~d}$ vacuum, provided (3.57) holds. In the equation above we have taken into account that the quadratic and quartic condensates are expected to be of the general form, cf. the discussion around (C.7),

$$
\mathcal{A} \propto l_{s}^{-1} e^{-c\left(l_{Y} / l_{s}\right)^{2}} ; \quad \mathcal{B} \propto l_{s}^{-2} e^{-2 c\left(l_{Y} / l_{s}\right)^{2}},
$$

up to proportionality constants of order one.

We have verified numerically, as a function of $\mathcal{A}^{2} / \mathcal{B}$, that all three eigenvalues of the Hessian of the potential are positive at the solution. I.e. the solution is a local minimum of the potential (3.48).

Flux quantization. The four-form flux is constrained to obey, ${ }^{11}$

$$
\frac{1}{l_{s}^{3}} \int_{\mathcal{C}_{A}} G \in \mathbb{Z}
$$

where $\left\{\mathcal{C}_{A} ; A=1, \ldots, h^{2,2}\right\}$ is a basis of integral four-cycles of the $\mathrm{CY}, \mathcal{C}_{A} \in H_{4}(Y, \mathbb{Z})$. From (3.25), (3.56), (3.59) we then obtain,

$$
n_{A} \propto g_{s}^{-1 / 4}\left(\frac{l_{Y}}{l_{s}}\right)^{4} e^{-c\left(l_{Y} / l_{s}\right)^{2}} \operatorname{vol}\left(\mathcal{C}_{A}\right)
$$

\footnotetext{
${ }^{10}$ If $\mathcal{B}>3 \mathcal{A}^{2} / 2$, we may also take the upper/lower sign in (3.56) for $\mathcal{A}$ positive/negative, respectively. Equation (3.57) is the weakest condition on the quartic condensate that is sufficient for consistency of the solution.

${ }^{11}$ The Page form corresponding to $G$ is given by $\hat{G}:=G-H \wedge \alpha$, which is closed. The difference between $G$ and $\hat{G}$ vanishes when integrated over four-cycles of $Y$.
} 
up to a proportionality constant of order one; $\operatorname{vol}\left(\mathcal{C}_{A}\right)$ is the volume of the four cycle $\mathcal{C}_{A}$ in units of $l_{Y}$, and $n_{A} \in \mathbb{Z}$. Since the string coupling can be tuned to obey $g_{s} \ll 1$ independently of the $l_{Y} / l_{s}$ ratio, $(3.61)$ can be solved for $\operatorname{vol}\left(\mathcal{C}_{A}\right)$ of order one, provided we take $n_{A}$ sufficiently close to each other. Given a set of flux quanta $n_{A}$, this equation fixes the Kähler moduli in units of $l_{Y}$; the overall CY volume is set by $l_{Y}$, which remains unconstrained.

Note that even if we allow for large flux quanta in order to solve the flux quantization constraint, it can be seen that higher-order flux corrections are subdominant in the $g_{s} \ll 1$ limit. Indeed the parameter that controls the size of these corrections is $\left|g_{s} G\right|$, which scales as $g_{s}^{3 / 4}$.

Similarly, the three-form flux is constrained to obey,

$$
\frac{1}{l_{s}^{2}} \int_{\mathcal{C}_{\alpha}} H \in \mathbb{Z}
$$

where $\left\{\mathcal{C}_{\alpha} ; A=1, \ldots, h^{2,1}\right\}$ is a basis of integral three-cycles of the CY, $\mathcal{C}_{\alpha} \in H_{3}(Y, \mathbb{Z})$. From (3.25) we can see that this equation constrains the periods of $\Omega$, and hence the complex-structure moduli of $Y$.

\section{Discussion}

We considered the effect of gravitino condensates from ALE instantons, in the context of a $4 \mathrm{~d}$ consistent truncation of IIA on CY in the presence of background flux. The $4 \mathrm{~d}$ theory admits de Sitter solutions, which are local minima of the potential (3.48), provided the quartic condensate has a positive sign, cf. (3.57). We do not know whether or not this is the case, as this would require knowledge of the explicit form of the zero modes of the Dirac operator in the $\tau=2$ ALE background. Clearly it would be crucial to construct these zero modes (which, to are knowledge, have never been explicitly computed), generalizing the calculations of $[29,30,40]$ to the second gravitational instanton in the ALE series.

The validity of the de Sitter solutions presented here requires the higher-order stringloop corrections in the $4 \mathrm{~d}$ action to be subdominant with respect to the ALE instanton contributions to the gravitino condensates. Since the latter do not depend on the string coupling, cf. (3.59), there is no obstruction to tuning $g_{s}$ to be sufficiently small, $g_{s} \ll 1$, in order for the string-loop corrections to be negligible with respect to the instanton contributions.

The $l_{Y} / l_{s}$ ratio can be tuned so that the condensates are of the order of the Einstein term in the $4 \mathrm{~d}$ action, thus dominating $4 \mathrm{~d}$ higher-order derivative corrections. This requires,

$$
l_{4 d}^{-2} \sim R_{\mathrm{dS}} \propto l_{s}^{-2} e^{-2 c\left(l_{Y} / l_{s}\right)^{2}}
$$

where we have taken (3.58) into account. Current cosmological data give,

$$
\frac{R_{\mathrm{dS}}}{M_{\mathrm{P}}^{2}} \sim\left(\frac{l_{s}}{l_{4 d}}\right)^{2} \sim 10^{-122} .
$$

From (4.1) we then obtain $l_{Y} / l_{s} \sim 10$ for $c$ of order one, cf. (2.13).

In addition to the higher-order derivative corrections, the $4 \mathrm{~d}$ effective action receives corrections at the two-derivative level, of the form $\left(l_{s} / l_{Y}\right)^{2 n}$ with $n \geq 1$. These come 
from a certain subset of the $10 \mathrm{~d}$ tree-level $\alpha^{\prime}$ corrections (string loops are subleading), which include the $R^{2}(\partial F)^{2}$ corrections of [36]. Given the $l_{Y} / l_{s}$ ratio derived above, these corrections will be of the order of one percent or less.

As is well known, the vacua computed within the framework of consistent truncations, such as the one constructed in the present paper, are susceptible to destabilization by modes that are truncated out of the spectrum. This is an issue that needs to be addressed before one can be confident of the validity of the vacua presented here. The stability issue is particularly important given the fact that, in the presence of a non-vanishing gravitino condensate, supersymmetry will generally be broken.

Ultimately, the scope of the path integral over metrics approach to quantum gravity is limited, since the $4 \mathrm{~d}$ gravity theory is non-renormalizable. Rather it should be thought of as an effective low-energy limit of string theory. A natural approach to gravitino condensation from the string/M-theory standpoint, would be to try to construct brane-instanton analogues of the four-dimensional gravitational instantons. The fermion condensates might then be computed along the lines of [50-52].

Another interesting direction would be to try to embed the consistent truncation of the present paper within the framework of $\mathcal{N}=24 \mathrm{~d}$ (gauged) supergravity. On general grounds [53], we expect the existence of a consistent truncation of a higher-dimensional supersymmetric theory to the bosonic sector of a supersymmetric lower-dimensional theory, to guarantee the existence of a consistent truncation to the full lower-dimensional theory. The condensate would then presumably be associated with certain gaugings of the $4 \mathrm{~d}$ theory.

\section{Acknowledgments}

We would like to thank Thomas Grimm and Kilian Mayer for useful correspondence.

\section{A Conventions}

As in [20], our spinor conventions are those listed in appendix A of [48], except that the $\mathrm{SU}(3)$-singlet spinor of the internal manifold is denoted $\eta$ here and corresponds to the $\eta_{+}$ of [48]. Moreover $\eta_{-}$of [48] corresponds to $\eta^{c}:=C \eta^{*}$ here.

Our conventions for the explicit $4 \mathrm{~d}$ spinor indices are as follows. A positive-, negativechirality $4 \mathrm{~d}$ Weyl spinor is indicated with a lower, upper spinor index respectively: $\theta_{\alpha}, \chi^{\alpha}$. We never raise or lower the spinor indices on spinors, so that the position unambiguously indicates the chirality. The $4 \mathrm{~d}$ gamma matrices, the charge conjugation and chirality matrices are decomposed into chiral blocks,

$$
\gamma_{\mu}=\left(\begin{array}{cc}
0 & \left(\gamma_{\mu}\right)_{\alpha \beta} \\
\left(\gamma_{\mu}\right)^{\alpha \beta} & 0
\end{array}\right) ; \quad C^{-1}=\left(\begin{array}{cc}
C^{\alpha \beta} & 0 \\
0 & C_{\alpha \beta}
\end{array}\right) ; \quad \gamma_{5}=\left(\begin{array}{cc}
\delta_{\alpha}^{\beta} & 0 \\
0 & -\delta^{\alpha}{ }_{\beta}
\end{array}\right)
$$

It is the "Pauli matrices" $\left(C^{-1} \gamma_{\mu_{1} \ldots \mu_{n}}\right)$ which act as Clebsch-Gordan coefficients between spinor bilinears and $n$-forms. For example, the structure of indices of the charge conjugation matrix reflects the fact that scalars can only be formed as spinor bilinears of Weyl spinors 
of the same chirality,

$$
v=\theta_{\alpha} C^{\alpha \beta} \chi_{\beta} ; \quad u=\theta^{\alpha} C_{\alpha \beta} \chi^{\beta} .
$$

As another example, the structure of indices of $C^{-1} \gamma_{\mu}$ reflects the fact that vectors can only be formed as spinor bilinears of Weyl spinors of opposite chirality,

$$
v_{\mu}=\theta^{\alpha}\left(C^{-1} \gamma_{\mu}\right)_{\alpha}{ }^{\beta} \chi_{\beta} ; \quad u_{\mu}=\theta_{\alpha}\left(C^{-1} \gamma_{\mu}\right)^{\alpha}{ }_{\beta} \chi^{\beta} .
$$

We also make use of the Fierz relation for two positive-chirality $4 \mathrm{~d}$ spinors,

$$
\theta_{\alpha} \chi_{\beta}=-\frac{1}{2}(\tilde{\theta} \chi) C_{\alpha \beta}-\frac{1}{8}\left(\tilde{\theta} \gamma_{\mu \nu} \chi\right)\left(\gamma^{\mu \nu} C\right)_{\alpha \beta},
$$

where $\tilde{\theta} \equiv \theta^{\operatorname{Tr}} C^{-1}$, and similarly for negative chirality.

The Hodge duality relations read,

$$
\frac{1}{(4-l) !} \varepsilon_{\mu_{1} \ldots \mu_{l}} \nu_{1 \ldots \nu_{4-l}} \gamma_{\nu_{1} \ldots \nu_{4-l}}=i^{s}(-1)^{\frac{1}{2} l(l-1)} \gamma_{\mu_{1} \ldots \mu_{l}} \gamma_{5}
$$

where $s=1,2$ for Lorentzian, Euclidean signature respectively. With explicit spinor indices in Euclidean signature we have,

$$
\frac{1}{2} \varepsilon_{\mu \nu \rho \sigma}\left(\gamma^{\rho \sigma}\right)_{\alpha}^{\beta}=\left(\gamma_{\mu \nu}\right)_{\alpha}^{\beta} ; \quad \frac{1}{2} \varepsilon_{\mu \nu \rho \sigma}\left(\gamma^{\rho \sigma}\right)^{\alpha}{ }_{\beta}=-\left(\gamma_{\mu \nu}\right)_{\beta}^{\alpha}
$$

In particular if $T_{\mu \nu}$ is a self-dual tensor, $\frac{1}{2} \varepsilon_{\mu \nu \rho \sigma} T^{\rho \sigma}=T_{\mu \nu}$, it follows that $T \cdot \gamma$ vanishes when acting on negative-chirality spinors,

$$
T^{\mu \nu}\left(\gamma_{\mu \nu}\right)_{\beta}^{\alpha}=0
$$

\section{B ALE instantons}

Asymptotically locally Euclidean (ALE) spaces, see e.g. [22] for a review, are noncompact self-dual gravitational instantons, i.e. their Riemann tensor obeys,

$$
\frac{1}{2} \varepsilon_{\mu \nu \rho \sigma} R_{\kappa \lambda}^{\rho \sigma}=R_{\kappa \lambda \mu \nu} .
$$

From the above and the identity $R_{[\kappa \lambda \mu] \nu}=0$, it follows that the ALE spaces are Ricci-flat,

$$
R_{\mu \nu}=0 .
$$

These spaces asymptote $S^{3} / \mathbb{Z}_{k+1}$ at spatial infinity, with $k \in \mathbb{N}$ (the case $k=0$ corresponds to $\mathbb{R}^{4}$ ). The simplest nontrivial example in this class is the EH space [28], which corresponds to $k=1$. Explicitly the metric reads,

$$
\mathrm{d} s^{2}=\mathrm{d} r^{2}\left(1-\frac{a^{4}}{r^{4}}\right)^{-1}+\frac{1}{4} r^{2}\left(\sigma_{1}^{2}+\sigma_{2}^{2}+\left(1-\frac{a^{4}}{r^{4}}\right) \sigma_{3}^{2}\right)
$$

where $a>0$ is an arbitrary constant, and,

$$
\sigma_{1}=\sin \psi \mathrm{d} \theta-\sin \theta \cos \psi \mathrm{d} \phi ; \quad \sigma_{2}=-\cos \psi \mathrm{d} \theta-\sin \theta \sin \psi \mathrm{d} \phi ; \quad \sigma_{3}=\mathrm{d} \psi+\cos \theta \mathrm{d} \phi
$$


For the coordinate ranges $a \leq r, 0 \leq \theta \leq \pi, 0 \leq \phi \leq 2 \pi, 0 \leq \psi \leq 2 \pi$, the manifold can be seen to be smooth with boundary given by $\mathbb{R P}^{3}=S^{3} / \mathbb{Z}_{2}$ at asymptotic infinity. (We would have an asymptotic $S^{3}$ if $0 \leq \psi \leq 4 \pi$ ). The Hirzebruch signature $\tau$ of a self-dual space is given by,

$$
\tau=\frac{1}{48 \pi^{2}} \int \mathrm{d} x^{4} \sqrt{g} R_{\kappa \lambda \mu \nu} R^{\kappa \lambda \mu \nu} \in \mathbb{N} .
$$

As can be verified using (B.3), the EH gravitational instanton is the ALE space with the smallest Hirzebruch signature, $\tau=1$. More generally it can be shown that $\tau=k$, with $k$ as given below eq. (B.2).

It is convenient to use a gauge in which not only the curvature but also the connection is self-dual [22],

$$
\omega_{a b}=\frac{1}{2} \varepsilon_{a b c d} \omega^{c d} .
$$

In this gauge the covariant derivative reduces to a simple derivative on negative chirality spinors,

$$
\nabla_{\mu} \theta^{\alpha}=\partial_{\mu} \theta^{\alpha}+\frac{1}{4} \omega_{\mu}^{a b}\left(\gamma_{a b}\right)^{\alpha}{ }_{\beta} \theta^{\beta}=\partial_{\mu} \theta^{\alpha},
$$

where in the last equality we took (A.7), (B.6) into account. It follows in particular that covariantly-constant negative-chirality spinors are just constant. We may therefore choose their basis $\theta_{(1)}^{\alpha}, \theta_{(2)}^{\alpha}$ as follows, in the chiral gamma-matrix basis of appendix A,

$$
\theta_{(1)}^{\alpha}=\left(\begin{array}{l}
1 \\
0
\end{array}\right) ; \quad \theta_{(2)}^{\alpha}=\left(\begin{array}{l}
0 \\
1
\end{array}\right) \text {. }
$$

The Atiyah-Patodi-Singer theorem for ALE spaces predicts an equal number of positiveand negative-chirality spinor zeromodes for the Dirac operator $\not>$ [29]. On the other hand we have,

$$
\begin{aligned}
\left(\not^{2} \theta\right)^{\alpha} & =\left(\nabla^{2} \theta+\gamma^{\mu \nu} \nabla_{\mu} \nabla_{\nu} \theta\right)^{\alpha} \\
& =\left(\nabla^{2} \theta+\frac{1}{8} R_{\mu \nu \rho \sigma} \gamma^{\mu \nu} \gamma^{\rho \sigma} \theta\right)^{\alpha} \\
& =\left(\nabla^{2} \theta\right)^{\alpha},
\end{aligned}
$$

where in the last equality we took (B.2) into account. It follows that negative-chirality zeromodes are (covariantly) constant, hence non-normalizable since the ALE space is noncompact. It thus follows from the index theorem that there are no (normalizable) spinor zeromodes of the Dirac operator.

For a spin-1 field ${ }^{12}$ the index theorem predicts that the number of positive-chirality zeromodes of the Dirac operator minus the number of negative-chirality zeromodes is equal to the Hirzebruch signature of the ALE space. We now have,

$$
\left(\not^{2} \phi\right)_{\alpha \beta}=\left(\nabla^{2} \phi\right)_{\alpha \beta}+\frac{1}{8} R_{\mu \nu \rho \sigma}\left(\gamma^{\mu \nu}\right)_{\alpha}^{\alpha^{\prime}}\left(\gamma^{\rho \sigma}\right)_{\beta}^{\beta^{\prime}} \phi_{\alpha^{\prime} \beta^{\prime}} ; \quad\left(\not \nabla^{2} \phi\right)^{\alpha \beta}=\left(\nabla^{2} \phi\right)^{\alpha \beta}
$$

\footnotetext{
${ }^{12}$ By a "spin-1 field" we understand a field transforming in the three-dimensional irreducible representation of the $s u(2)$ algebra. It can be thought of as a field with two symmetric spinor indices of the same chirality, $\phi_{\alpha \beta}=\phi_{\beta \alpha}$ (positive chirality) or $\phi^{\alpha \beta}=\phi^{\beta \alpha}$ (negative chirality).
} 
where in the second equation we took (A.7) into account. By the same argument as before, it follows that $\phi^{\alpha \beta}$ is covariantly constant, hence non-renormalizable. Therefore there are no spin-1 fields of negative chirality. By the index theorem it follows that there are $\tau$ spin- 1 zeromodes of positive chirality (i.e. one zeromode for the EH space).

A massless gravitino $\psi_{\mu}$ is also a zeromode of the Dirac operator $\not$, in the gauge $\gamma^{\mu} \psi_{\mu}=0$. By a similar argument as before, there are $2 \tau$ spin-3/2 zeromodes of positive chirality. These can be constructed as follows,

$$
\psi_{(i) \mu \alpha}=\phi_{\alpha \beta} \theta_{(i)}^{\gamma}\left(C^{-1} \gamma_{\mu}\right)_{\gamma}{ }^{\beta} ; i=1,2,
$$

where $\theta_{(i)}$ are the covariantly-constant spinors of (B.8), and $\phi_{\alpha \beta}$ are the positive-chirality spin-1 zeromodes of (B.10). Indeed we verify that the $\psi_{(i) \mu \alpha}$ are traceless,

$$
\left(\gamma^{\mu}\right)^{\alpha \beta} \psi_{(i) \mu \alpha}=0 ; i=1,2,
$$

as follows from (B.11) and the identity $\left(C^{-1} \gamma_{\mu}\right)_{(\gamma}{ }^{\beta}\left(C^{-1} \gamma^{\mu}\right)_{\delta)}{ }^{\alpha}=0$. Moreover they obey the zeromode equation,

$$
\left(\nabla^{2} \psi_{\mu}\right)_{\alpha}=\left(\nabla^{2} \psi_{\mu}+\frac{1}{2} \gamma^{\rho \sigma} R_{\mu \rho \sigma}{ }^{\nu} \psi_{\nu}\right)_{\alpha}=0
$$

where we used (B.11) and the Hodge duality relations (A.5).

\section{Gravitino condensates in $4 \mathrm{~d} \mathcal{N}=1$ supergravity}

Within the context of $4 \mathrm{~d} \mathcal{N}=1$ supergravity, the condensate $\left\langle\psi^{\mu} \psi_{\mu}^{\prime}\right\rangle$ was shown in [29] to be proportional to the zeromode bilinear. From (B.11) we get,

$$
\tilde{\psi}_{(1) \mu} \psi_{(2)}^{\mu}=f ; \quad f:=2 C^{\alpha \beta} \phi_{\beta \gamma} C^{\gamma \delta} \phi_{\delta \alpha}
$$

where $f$ is a positive function on the ALE space, and we have normalized $\tilde{\theta}_{(1)} \theta_{(2)}=1$. In deriving the above we have noted that $\phi_{\alpha \gamma} C^{\gamma \delta} \phi_{\delta \beta}$ is antisymmetric in its free indices, therefore it is necessarily proportional to $C_{\alpha \beta}$, since there is a unique scalar in the decomposition of the antisymmetric product of two spinors of positive chirality. For the EH space, cf. (B.3), $f$ can be given explicitly as in [30],

$$
f=16\left(\frac{a}{r}\right)^{8} .
$$

The zeromode normalization can thus be inferred from,

$$
\int \mathrm{d}^{4} x \sqrt{g} \tilde{\psi}_{(1) \mu} \psi_{(2)}^{\mu}=\frac{1}{2} \operatorname{Vol}\left(S^{3}\right) \int_{a}^{\infty} \mathrm{d} r r^{3} f=4 \pi^{2} a^{4},
$$

where the "spherical" coordinates in (B.3) are related to the cartesian coordinates $x^{\mu}$ in the usual way, except that antipodal points on $S^{3}$ are identified, see below (B.4).

To calculate the gravitino bilinear we follow [30] who adopt the prescription of [21] for the functional integration over metrics. As shown explicitly in [30] in the case of $4 \mathrm{~d} \mathcal{N}=1$ 
supergravity, expanding the action around the EH instanton saddle point and performing the Gaussian integrations, the one-loop determinants from all massive modes cancel out thanks to supersymmetry. One is then left with the integration over zeromodes. The latter reduces to an integration over the instanton size,

$$
\begin{aligned}
\left\langle\tilde{\psi}_{\mu} \psi^{\mu}\right\rangle & =\text { const. } M_{\mathrm{P}} e^{-S_{0}} \int \mathrm{d} a a^{5} \tilde{\psi}_{(1) \mu} \psi_{(2)}^{\mu} \\
& =\text { const. } M_{\mathrm{P}} e^{-S_{0}} \int \mathrm{d} a a^{5}\left(\frac{a}{r}\right)^{8} a^{-4},
\end{aligned}
$$

where we have used (C.1), (C.2) and normalized $\psi_{\mu} \rightarrow \psi_{\mu} /\left(2 \pi a^{2}\right)$, cf. (C.3); the remaining power of $a$ comes from the Jacobian of the transformation from the integration over metric zeromodes to the integration over the instanton moduli.

The integration in (C.4) would seem to depend on the spacetime position, since $a$ is bounded above by the radial distance $r$. In order to overcome this problem, [30] performs a coordinate transformation,

$$
\tilde{x}^{\mu}=\frac{u}{r} x^{\mu} ; \quad u:=r \sqrt{1-\left(\frac{a}{r}\right)^{4}},
$$

which has the effect of changing the radial coordinate from $r \geq a$ to $u \geq 0$. We can then rewrite (C.4) as follows,

$$
\left\langle\tilde{\psi}_{\mu} \psi^{\mu}\right\rangle=\text { const. } M_{\mathrm{P}} e^{-S_{0}} \int_{0}^{\infty} \mathrm{d} a a^{9}\left(u^{2}+\sqrt{4 a^{4}+u^{4}}\right)^{-4} .
$$

This integral diverges for $a \rightarrow \infty$ at fixed $u$. In contrast, the same calculation for the gravitino fieldstrength bilinear $\left\langle\left(\nabla_{[\mu} \psi_{\nu]}\right)^{2}\right\rangle$ yields a finite result [30]. This is due to the fact that the two derivatives bring about an extra $\left(u / r^{2}\right)^{2}$ factor compared to the integrand in (C.6), which contributes an extra $a^{-4}$ factor in the $a \rightarrow \infty$ limit. However even this finite result seems to rely on the coordinate system (C.5). This does not seem satisfactory: for diffeomorphism invariance to be respected, the result should be independent of the coordinate system used for its calculation.

One may argue that the divergence/ambiguity encountered is not surprising since the $4 \mathrm{~d}$ theory is nonrenormalizable and should anyway be thought of as an effective low-energy limit of string theory. On general grounds, at one loop in the gravitational coupling, one expects a gravitational instanton contribution to the condensate of the form,

$$
\left\langle\tilde{\psi}_{\mu} \psi^{\mu}\right\rangle \propto M_{\mathrm{P}} e^{-S_{0}} \propto l_{s}^{-1} e^{-c\left(l_{Y} / l_{s}\right)^{2}},
$$

up to proportionality constants of order one, where in the second proportionality we have taken (2.8), (2.13) into account. Similarly, the quartic gravitino condensate receives contributions from the ALE with $\tau=2$ and scales as the square of the bilinear condensate above.

Open Access. This article is distributed under the terms of the Creative Commons Attribution License (CC-BY 4.0), which permits any use, distribution and reproduction in any medium, provided the original author(s) and source are credited. 


\section{References}

[1] U.H. Danielsson and T. Van Riet, What if string theory has no de Sitter vacua?, Int. J. Mod. Phys. D 27 (2018) 1830007 [arXiv:1804.01120] [INSPIRE].

[2] E. Palti, The swampland: introduction and review, Fortsch. Phys. 67 (2019) 1900037 [arXiv: 1903.06239] [INSPIRE].

[3] B. de Wit, D.J. Smit and N.D. Hari Dass, Residual supersymmetry of compactified D $=10$ supergravity, Nucl. Phys. B 283 (1987) 165 [INSPIRE].

[4] J.M. Maldacena and C. Núñez, Supergravity description of field theories on curved manifolds and a no go theorem, Int. J. Mod. Phys. A 16 (2001) 822 [hep-th/0007018] [INSPIRE].

[5] C. Córdova, G.B. De Luca and A. Tomasiello, Classical de Sitter solutions of 10-dimensional supergravity, Phys. Rev. Lett. 122 (2019) 091601 [arXiv: 1812.04147] [INSPIRE].

[6] D. Andriot, New constraints on classical de Sitter: flirting with the swampland, Fortsch. Phys. 67 (2019) 1800103 [arXiv:1807.09698] [INSPIRE].

[7] N. Cribiori and D. Junghans, No classical (anti-)de Sitter solutions with O8-planes, Phys. Lett. B 793 (2019) 54 [arXiv:1902.08209] [INSPIRE].

[8] D. Andriot, Open problems on classical de Sitter solutions, arXiv:1902.10093 [INSPIRE].

[9] V.A. Novikov, M.A. Shifman, A.I. Vainshtein and V.I. Zakharov, Instanton effects in supersymmetric theories, Nucl. Phys. B 229 (1983) 407 [InSPIRE].

[10] M. Dine, R. Rohm, N. Seiberg and E. Witten, Gluino condensation in superstring models, Phys. Lett. B 156 (1985) 55 [inSPIRE].

[11] J.P. Derendinger, L.E. Ibáñez and H.P. Nilles, On the low-energy $d=4, N=1$ supergravity theory extracted from the $d=10, N=1$ superstring, Phys. Lett. B 155 (1985) 65 [INSPIRE].

[12] G. Lopes Cardoso, G. Curio, G. Dall'Agata and D. Lüst, Heterotic string theory on non-Kähler manifolds with $H$ flux and gaugino condensate, Fortsch. Phys. 52 (2004) 483 [hep-th/0310021] [INSPIRE].

[13] J.-P. Derendinger, C. Kounnas and P.M. Petropoulos, Gaugino condensates and fluxes in $N=1$ effective superpotentials, Nucl. Phys. B 747 (2006) 190 [hep-th/0601005] [InSPIRE].

[14] P. Manousselis, N. Prezas and G. Zoupanos, Supersymmetric compactifications of heterotic strings with fluxes and condensates, Nucl. Phys. B 739 (2006) 85 [hep-th/0511122] [INSPIRE].

[15] A. Chatzistavrakidis, O. Lechtenfeld and A.D. Popov, Nearly Kähler heterotic compactifications with fermion condensates, JHEP 04 (2012) 114 [arXiv:1202.1278] [INSPIRE].

[16] K.-P. Gemmer and O. Lechtenfeld, Heterotic $G_{2}$-manifold compactifications with fluxes and fermionic condensates, JHEP 11 (2013) 182 [arXiv:1308.1955] [INSPIRE].

[17] R. Minasian, M. Petrini and E.E. Svanes, On heterotic vacua with fermionic expectation values, Fortsch. Phys. 65 (2017) 1700010 [arXiv: 1702.01156] [INSPIRE].

[18] C. Quigley, Gaugino condensation and the cosmological constant, JHEP 06 (2015) 104 [arXiv: 1504.00652] [INSPIRE].

[19] B. Souères and D. Tsimpis, De Sitter space from dilatino condensates in (massive) IIA, Phys. Rev. D 97 (2018) 046005 [arXiv:1712.07169] [INSPIRE]. 
[20] R. Terrisse and D. Tsimpis, Consistent truncation with dilatino condensation on nearly Kähler and Calabi-Yau manifolds, JHEP 02 (2019) 088 [arXiv: 1810.06344] [INSPIRE].

[21] G.W. Gibbons, S.W. Hawking and M.J. Perry, Path integrals and the indefiniteness of the gravitational action, Nucl. Phys. B 138 (1978) 141 [INSPIRE].

[22] T. Eguchi, P.B. Gilkey and A.J. Hanson, Gravitation, gauge theories and differential geometry, Phys. Rept. 66 (1980) 213 [INSPIRE].

[23] E. Witten, Dynamical breaking of supersymmetry, Nucl. Phys. B 188 (1981) 513 [INSPIRE].

[24] R.M. Schon and S.-T. Yau, Proof of the positive action conjecture in quantum relativity, Phys. Rev. Lett. 42 (1979) 547 [INSPIRE].

[25] R. Schon and S.-T. Yau, On the proof of the positive mass conjecture in general relativity, Commun. Math. Phys. 65 (1979) 45 [INSPIRE].

[26] R. Schon and S.-T. Yau, Proof of the positive mass theorem. 2, Commun. Math. Phys. 79 (1981) 231 [INSPIRE].

[27] E. Witten, A simple proof of the positive energy theorem, Commun. Math. Phys. 80 (1981) 381 [INSPIRE].

[28] T. Eguchi and A.J. Hanson, Selfdual solutions to Euclidean gravity, Annals Phys. 120 (1979) 82 [INSPIRE].

[29] S.W. Hawking and C.N. Pope, Symmetry breaking by instantons in supergravity, Nucl. Phys. B 146 (1978) 381 [inSPIRE].

[30] K. Konishi, N. Magnoli and H. Panagopoulos, Spontaneous breaking of local supersymmetry by gravitational instantons, Nucl. Phys. B 309 (1988) 201 [INSPIRE].

[31] I. Antoniadis, S. Ferrara, R. Minasian and K.S. Narain, $R^{4}$ couplings in $M$ and type-II theories on Calabi-Yau spaces, Nucl. Phys. B 507 (1997) 571 [hep-th/9707013] [InSPIRE].

[32] T.W. Grimm, K. Mayer and M. Weissenbacher, Higher derivatives in type II and M-theory on Calabi-Yau threefolds, JHEP 02 (2018) 127 [arXiv: 1702.08404] [INSPIRE].

[33] M. Weissenbacher, F-theory vacua and $\alpha^{\prime}$-corrections, arXiv:1901.04758 [INSPIRE].

[34] D.J. Gross and J.H. Sloan, The quartic effective action for the heterotic string, Nucl. Phys. B 291 (1987) 41 [INSPIRE].

[35] J.T. Liu and R. Minasian, Higher-derivative couplings in string theory: dualities and the B-field, Nucl. Phys. B 874 (2013) 413 [arXiv: 1304.3137] [inSPIRE].

[36] G. Policastro and D. Tsimpis, $R^{4}$, purified, Class. Quant. Grav. 23 (2006) 4753 [hep-th/0603165] [INSPIRE].

[37] M. Haack and J.U. Kang, One-loop Einstein-Hilbert term in minimally supersymmetric type IIB orientifolds, JHEP 02 (2016) 160 [arXiv:1511.03957] [INSPIRE].

[38] S. Katmadas and R. Minasian, $N=2$ higher-derivative couplings from strings, JHEP 02 (2014) 093 [arXiv: 1311.4797] [INSPIRE].

[39] D. Nemeschansky and A. Sen, Conformal invariance of supersymmetric $\sigma$ models on Calabi-Yau manifolds, Phys. Lett. B 178 (1986) 365 [INSPIRE].

[40] M. Bianchi, F. Fucito, G.C. Rossi and M. Martellini, ALE instantons in string effective theory, Nucl. Phys. B 440 (1995) 129 [hep-th/9409037] [INSPIRE]. 
[41] K. Konishi, N. Magnoli and H. Panagopoulos, Generation of mass hierarchies and gravitational instanton induced supersymmetry breaking, Nucl. Phys. B 323 (1989) 441 [INSPIRE].

[42] F. Giani and M. Pernici, $N=2$ supergravity in ten-dimensions, Phys. Rev. D 30 (1984) 325 [INSPIRE].

[43] I.C.G. Campbell and P.C. West, $N=2, D=10$ nonchiral supergravity and its spontaneous compactification, Nucl. Phys. B 243 (1984) 112 [INSPIRE].

[44] M. Huq and M.A. Namazie, Kaluza-Klein supergravity in ten-dimensions, Class. Quant. Grav. 2 (1985) 293 [Erratum ibid. 2 (1985) 597] [INSPIRE].

[45] L.J. Romans, Massive $N=2 a$ supergravity in ten-dimensions, Phys. Lett. B 169 (1986) 374 [INSPIRE].

[46] P.S. Howe, N.D. Lambert and P.C. West, A new massive type IIA supergravity from compactification, Phys. Lett. B 416 (1998) 303 [hep-th/9707139] [INSPIRE].

[47] D. Tsimpis, Massive IIA supergravities, JHEP 10 (2005) 057 [hep-th/0508214] [INSPIRE].

[48] D. Lüst and D. Tsimpis, Supersymmetric AdS 4 compactifications of IIA supergravity, JHEP 02 (2005) 027 [hep-th/0412250] [INSPIRE].

[49] E.A. Bergshoeff, J. Hartong, A. Ploegh, J. Rosseel and D. Van den Bleeken, Pseudo-supersymmetry and a tale of alternate realities, JHEP 07 (2007) 067 [arXiv: 0704.3559] [INSPIRE].

[50] K. Becker, M. Becker and A. Strominger, Five-branes, membranes and nonperturbative string theory, Nucl. Phys. B 456 (1995) 130 [hep-th/9507158] [INSPIRE].

[51] J.A. Harvey and G.W. Moore, Superpotentials and membrane instantons, hep-th/9907026 [INSPIRE].

[52] D. Tsimpis, Fivebrane instantons and Calabi-Yau fourfolds with flux, JHEP 03 (2007) 099 [hep-th/0701287] [INSPIRE].

[53] M. Cvetič, H. Lü and C.N. Pope, Consistent Kaluza-Klein sphere reductions, Phys. Rev. D 62 (2000) 064028 [hep-th/0003286] [INSPIRE]. 